\title{
Guide to SAFT-UT Principles and Conventions
}

T. E. Hall

October 1987

Prepared for

Sandia National Laboratories

under a Related Services Agreement with

the U.S. Department of Energy

under Contract DE-AC06-76RLO 1830

Pacific Northwest Laboratory

Operated for the U.S. Department of Energy

by Battelle Memorial Institute 


\title{
DISCLAIMER
}

This report was prepared as an account of work sponsored by an agency of the United States Government. Neither the United States Government nor any agency thereof, nor Battelle Memorial Institute, nor any of their employees, makes any warranty, expressed or implied, or assumes any legal liability or responsibility for the accuracy, completeness, or usefulness of any information, apparatus, product, or process disclosed, or represents that its use would not infringe privately owned rights. Reference herein to any specific commercial product, process, or service by trade name, trademark, manufacturer, or otherwise, does not necessarily constitute or imply its endorsement, recommendation, or favoring by the United States Government of any agency thereof, or Battelle Memorial Institute. The views and opinions of authors expressed herein do not necessarly state or reflect those of the United States Government or any agency thereof, or Battelle Memorial Institute.

\author{
PACIFIC NORTHWEST LABORATORY \\ operated by \\ BATTELLE MEMORIAL INSTITUTE \\ for the \\ UNITED STATES DEPARTMENT OF ENERGY \\ under Contract DE-AC06-76RLO 1830
}

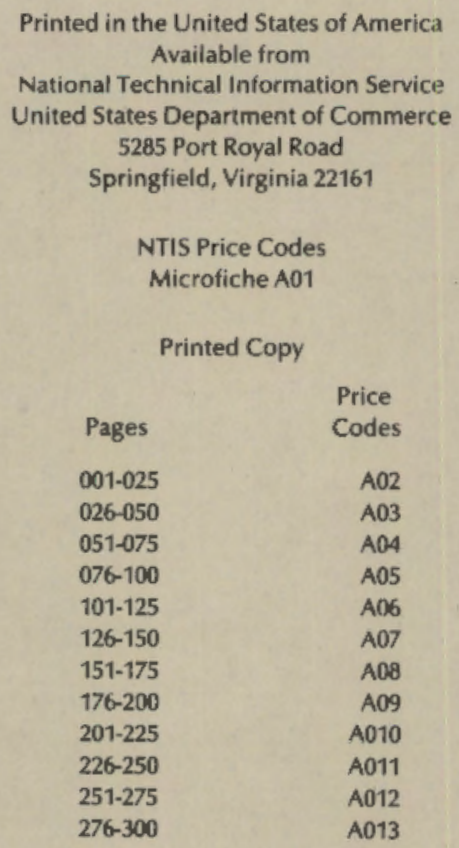




\section{SAFT-UT UTILITIES \\ GUIDE TO SAFT-UT PRINCIPLES AND CONVENTIONS}

T. E. Hall

October 1987

Prepared for

Sandia National Laboratories under a Related Services Agreement with the U.S. Department of Energy under Contract DE-ACO6-76RLO 1830

Pacific Northwest Laboratory Richland, Washington 99352 



\section{ABSTRACT}

This document is the first in a set of three reference documents pertaining to the SAFT-UT UTILITIES. The second document, the INTERFACE AND INSTALLATION GUIDE, describes the steps necessary to install the SAFT utilities and RealTime Processor on a VAX class computer running the VMS operating system. It also describes in detail the assumed data file format to accommodate adaptation of user data files to the SAFT-UT standard format. The third manual is the REFERENCE MANUAL, which describes in detail the operation of each utility available to the user.

This guide, the GUIDE TO SAFT-UT PRINCIPLES AND CONVENTIONS, is intended to provide the user with an overall background for practical implementation of SAFT-UT and the associated software utilities. It is not intended to exhaustively cover each related $i$ tem, but rather to supply information from theory and experience that will assist the serious user in exploiting the robust nature of the SAFT technology.

Chapter 1 gives an introductory overview from a historical perspective, emphasizing the need to maintain data integrity throughout the SAFT-UT imaging process.

Chapter 2 includes a theoretical description of the three SAFT-UT configurations currently being supported (single transducer pulse-echo, TSAFT, and TSAFT-2).

A general description of the software tools is given in chapter 3 . It describes the major utilities and the general use of each one. UT.

Chapter 4 deals with the system resolution and sampling demands of SAFT-

A discussion of the strengths and limitations of the SAFT technique is provided in chapter 5 .

The scanning conventions assumed throughout the implementation of the SAFT-UT utilities are detailed in chapter 6.

Chapter 7 lists an exhaustive supply of references for the user desiring to investigate the SAFT technology further. 


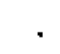

$\because$

, 


\section{CONTENTS}

1.0 INTRODUCTION

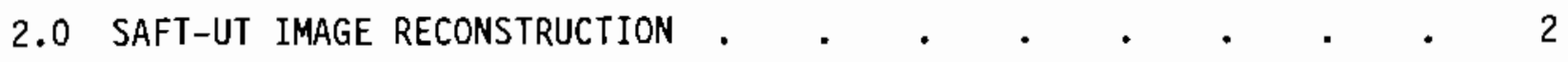

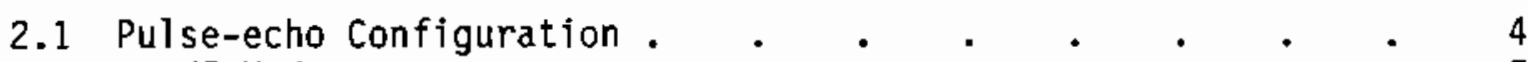

2.2 TSAFT Mode.$\quad \cdot \quad \cdot \quad \cdot \quad \cdot \quad \cdot \quad \cdot \quad \cdot \quad \cdot \quad \cdot 7$

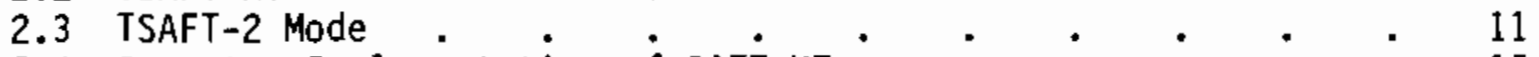

2.4 Computer Implementation of SAFT-UT : $: \quad \cdot \quad \cdot \quad: \quad \cdot \quad 15$

3.0 SAFT-UT DATA COLLECTION, PROCESSING, AND ANALYSIS • • . . 18

3.1 Primary Data Acquisition Considerations . . . . . . 18

3.2 Aperture Definition. . . . . . . . . . 20

3.3 Preprocessing Tools . . . . . . . . . . 22

3.4 Processing the Data File . . . . . . . . 25

3.5 Display and Analysis of the Final Image . . . . . . 27

4.0 SAMPLING AND RESOLUTION REQUIREMENTS .

4.1 System Resolution . . . . . . . . . 30

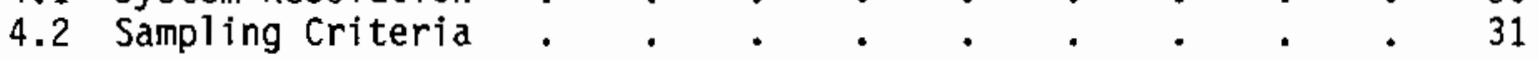

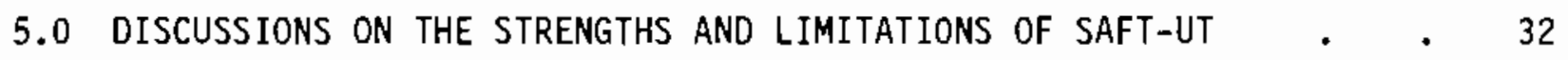

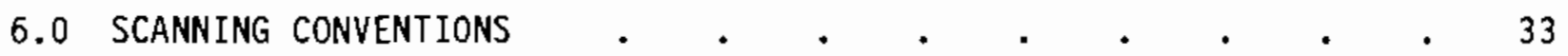

7.0 WHERE TO GO FOR MORE INFORMATION (REFERENCES) . . . . . . 35

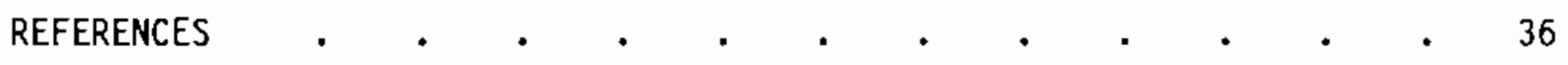




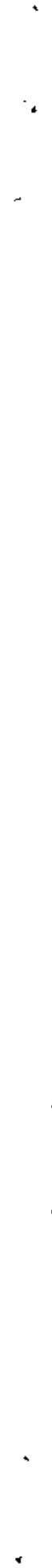




\section{FIGURES}

1. Sound Paths for the Single Transducer Pulse-Echo

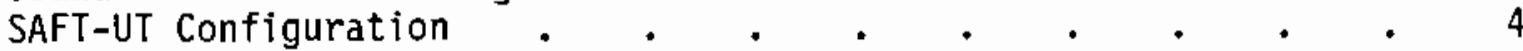

2. Curves Representing the Range for the P1-P1 Sound Path in the SAFT-UT Pulse-Echo Mode . . . . . . . 5

3. Curves Representing the Range for the P1-P3-P2 Sound Path in the SAFT-UT Pulse-Echo Mode . . . . . . 6

4. B-scan Side-View and B-scan End-View SAFT-UT PulseEcho Image of a Vertically Oriented Specular Sawcut in a Stainless Steel Coupon . . . . . . . . . 7

5. Four Propagation Paths for Ultrasonic Energy Produced

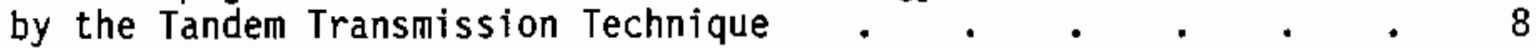

6. Two Propagation Paths ( $A$ and $B$ ) from the Transmitter

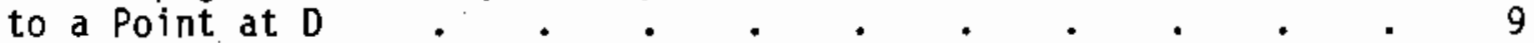

7. Two Propagation Paths ( $A$ and $B$ ) from a Point at $D$ to

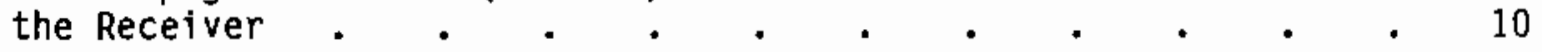

8. Path 2 Curves of the Total Center Ray Transmitter Sound Path for a Point Reflector at Depth D . $\quad$ • $\quad$ • $\quad$ • 11

9. B-scan Side-View and B-scan End-View TSAFT Image of a Vertically Oriented Specular Sawcut in a Stainless Steel Coupon . $\quad . \quad$. . . . . . . . . . . . 12

10a. TSAFT Center Ray Paths for Point Reflectors $P_{1}$ and $P_{2}$. $\quad$. 13

10b. TSAFT-2 Center Ray Paths for Point Reflectors $P_{1}$ and $P_{2}$. $\quad$ - 13

11. 'Comparison' Presentation of the TSAFT (top) and TSAFT-2 (bottom) Images of Two Opposing Vertical

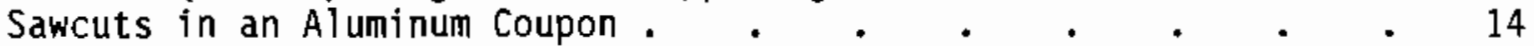

12. TSAFT-2 Image (B-scan Side-View and B-scan End-View) of Fabrication Defects in Carbon Steel Specimen . . . . . 15

13. Pictorial Representation of the SAFT-UT Coherent Summation Algorithm . 
14. SAFT-UT Host Computer Software Configuration . . . . 19

15. Normal Beam 3-Dimensional Synthetic Aperture Geometry . . . 20

16. Normal Beam 3-Dimensional Synthetic Aperture Geometry with a Finite "Entry Point Diameter" (D) Specified . . . 22

17. Normal Beam 3-Dimensional Synthetic Aperture Geometry with the THROUGH Option Selected and an "Entry Point

Diameter" of D. . . . . . . . . . . 23

18. Normal Beam 3-Dimensional Synthetic Aperture Geometry with the DOUGHNUT Option Selected and an "Entry Point

Diameter" of D . . . . . . . . . . . . 24

19. SAFT-UT Scan Convention . . . . . . . . . . . . 34 


\subsection{INTRODUCTION}

Since the mid-1970's the Nuclear Regulatory Commission has supported development of the Synthetic Aperture Focusing Technique for applications in ultrasonic inspection (SAFT-UT). Specifically, the inspection of critical components in nuclear reactors has been the major application emphasis. Research began at the University of Michigan, which included laboratory adapta- . tion of SAFT algorithms to the ultrasonic realm. An extensive laboratory oriented software library was developed on a Digital Equipment Corporation VAX-11/780 running the UNIX operating system.

In 1983 the research effort was transferred to PNL to develop a fieldable system based on the technology developed at the University of Michigan. At PNL the effort was focused on accelerating the computation process, streamlining the software package, and reducing the physical size of the hardware involved. "Portability" became the key word in terms of both the hardware and software.

A major objective was to offer a practical and fieldable system to the commercial sector. This process many times is referred to as "technology transfer". It was considered important to the success of technology transfer to provide a user friendly software package, and offer the ability of integrating the developed SAFT processing accelerator (Real-Time SAFT Processor) on the user's host computer.

The result of the PNL effort is a VAX VMS-based utility set that facilitates use of a robust ultrasonic imaging technique. It is the desire of the personnel involved at PNL, that the tools provided will stimulate further SAFT development and implementations. With knowledgeable implementation of SAFT in ultrasonic applications, quality imaging will result, and the reputation of SAFT capabilities will be promoted.

The integrity of the original data base is one of the most critical concepts that directly affects the output quality of any imaging system. Maintaining this integrity includes ensuring that the data is of high quality and is controlled by a number of parameters. The signal-to-noise ratio needs to be optimized, within reason, for a given test. The mechanical components such as the scanner must be of sufficient precision as to not corrupt the data processing. The analog amplifiers must be linear and of sufficient gain so that the signal from the echo of interest has significant dynamic range. The digitizer must reliably provide an accurate and linear digital representation of the supplied analog signal. Also the digitization rate must be high enough to fully characterize the frequency components of the returned signals that are of interest.

In addition the user must be careful to document the parameters that are relevant to subsequent signal processing and interpretation. This is particularly true when the data is collected for the purpose of SAFT processing. The SAFT-UT data files store the parameters relative to the inspection in the 
first portion of each data file. This ensures that as long as the data file is archived, a record of the specific scan parameters is also available. However, this parameter block is only valid if care has been taken to complete the information for input to the SAFT-UT utilities. It is recommended that a menu driven data acquisition system be implemented to assist the user in supplying accurate and complete parameter information.

This document provides the information necessary to ensure the integrity of the data collected and the parameter information associated with that data file. It has been our experience at PNL that the robust nature of the SAFT technique and algorithm provides quality ultrasonic imaging, and that the majority of erroneous interpretations in the past have been due to errors in the data collection phase of the process. It is in the interest of the future of SAFT-UT technology that the principles and conventions of this utility set is clearly communicated so erroneous results may be avoided.

The following chapters include a general overview of the SAFT theory for the current SAFT-UT algorithms, procedures related to data collection and analysis, sampling recommendations, a description of the scan conventions assumed throughout the SAFT-UT utilities, and finally an extensive reference list for those wishing to investigate this technology further. 


\subsection{SAFT-UT IMAGE RECONSTRUCTION}

"Synthetic aperture focusing" refers to a process in which the focal properties of a large-aperture focused transducer are synthetically generated from data collected over a large area using a small transducer with a divergent sound field. The processing required to focus this collection of data has been called beam-forming, coherent summation, or synthetic aperture processing.

Inherently, SAFT has an advantage over physical focusing techniques in that the resulting image is a full-volume focused characterization of the inspected area. Traditional physical focusing techniques provide focused data only at the depth of focus of the lens. For the typical pulse-echo data collection scheme used with SAFT-UT, a focused transducer is positioned with the focal point located at the surface of the part to be inspected. This configuration is used to produce a broad, divergent ultrasonic beam in the object under test. Alternatively, a small diameter contact transducer may be used to generate a divergent beam in the specimen material. As the transducer is scanned over the surface of the object, the A-scan record ( $\mathrm{rf}$ waveform) is digitized for each position of the transducer. Each reflector produces a collection of echoes in the A-scan records. If the reflector is an elementary single point reflector, then the collection of echoes will form a hyperbolic surface within the data-set volume. The shape of the hyperboloid is determined by the depth of the reflector in the test object and the velocity of sound in the material under test. This relationship between echo location in the series of A-scans and the actual location of the reflectors within the test object makes it possible to reconstruct a high-resolution image from the acquired signals.

If the scanning and surface geometries are well known, it is possible to accurately predict the shape of the locus of echoes for each point within the test object. The process of coherent summation for each image point involves shifting a locus of A-scans, within a regional aperture, by predicted time delays and summing the shifted A-scans. This process may also be viewed as performing a spatial matched filter operation for each point within the volume to be imaged. Each element is then averaged by the number of points that were summed to produce the final processed value. If the particular location correlates with the elementary point response hyperboloid, then the values summed will be in phase and produce a high-amplitude result. If the location does not correlate with the predicted response, then destructive interference will take place and the spatial average will result in a low amplitude.

A description of the current configurations implemented in the SAFT-UT software will follow. First the single transducer, pulse-echo configuration, is described; with the dual transducer tandem configurations, TSAFT and TSAFT2, described subsequently. 


\subsection{PULSE-ECHO CONFIGURATION}

The principles of the elementary single-transducer SAFT-UT pulse-echo configuration are essential to comprehend in order to accurately interpret results of the SAFT-UT process and to understand the more complex multipletransducer configurations. SAFT-UT pulse-echo is a single-transducer sourcereceiver scanning configuration (i.e., the same transducer that generates the sound field in the object space also receives the return echo).

In thin materials, where reflections from the back surface need to be considered, multiple sound paths may contribute to the reconstructed image. In the SAFT-UT pulse-echo configuration there are two of these candidate propagation paths that are dominant when observing generally vertical oriented defects. These are illustrated in Figure 1. The part as shown has a thickness of T with an elementary point reflector at depth $D$ from the scanned surface. The transducer scanned has its focus located at the surface, and in general has a non-zero incident angle.

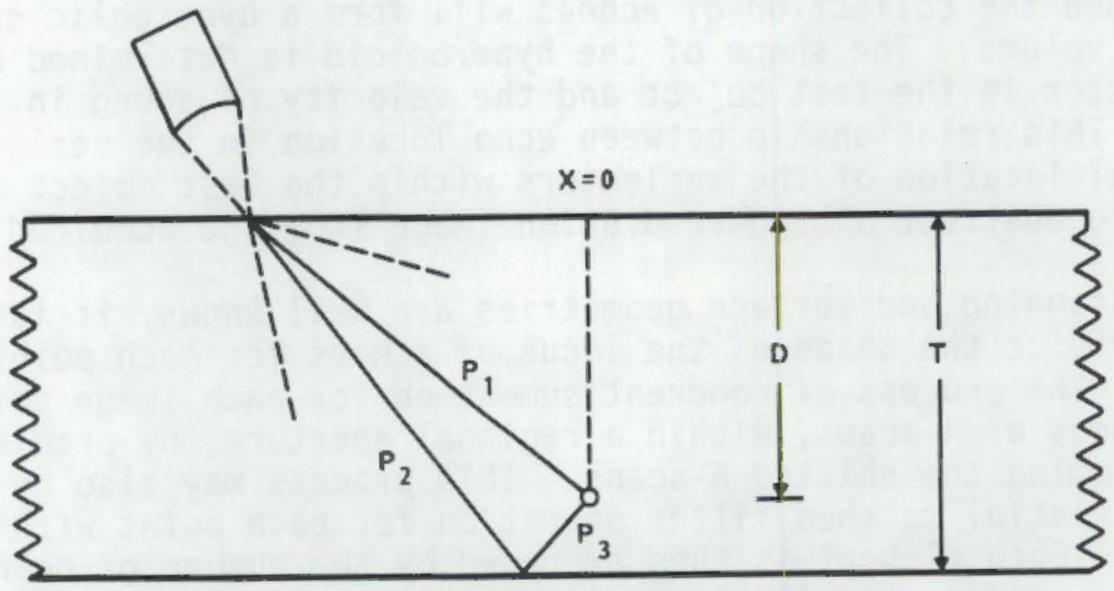

FIGURE 1. Sound Paths for the Single Transducer Pulse-Echo SAFT-UTT Configuration

The first path to be considered is the direct path $\left(P_{1}-P_{1}\right)$ from the beam entry point to the reflector and back to the entry point, without intersecting the far surface. Eq. 1 shows the computation of the direct sound path length.

$$
P=\left[D^{2}+x^{2}\right]^{1 / 2}
$$




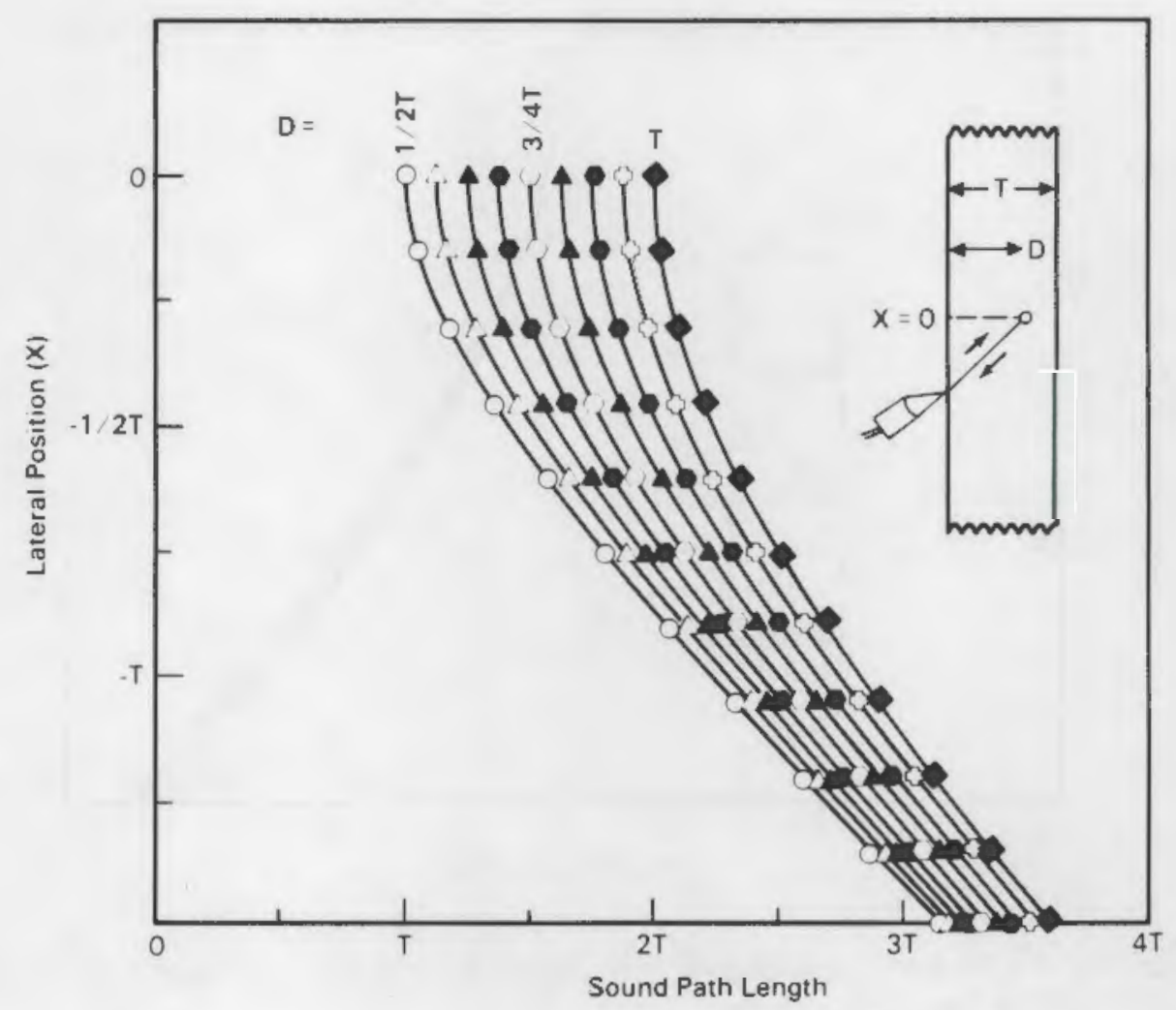

FIGURE 2. Curves Representing the Range for the P1-P1 Sound Path in the SAFT-UT Pulse-Echo Mode

When this equation is observed graphically (Figure 2), it is apparent that data received along this path will be deterministic and represent accurately and uniquely lateral position and depth information. This direct path represents the sometimes elusive 'tip' signal commonly referred to when imaging vertical-oriented defects in piping such as IGSCC. Or in thick material, this sound path may represent the only energy received from a volumetric type reflector in the specimen.

The second path of interest $\left(P_{1}-P_{2}-P_{3}\right)$ is the propagation path from the entry point to the far surface to the object and back to the transducer. This sound path length may be represented by Eq. 2 .

$$
P=\left(D^{2}+x^{2}\right)^{1 / 2}+\left[(2 T-D)^{2}+x^{2}\right]^{1 / 2}
$$

Figure 3 shows the family of curves generated when solving Eq. 2, while discretely varying depth $D$. A definite ambiguity is apparent when observing this diagram. Very little unique object depth information is available from reconstruction of this sound path, and as a result the time-of-flight of the reflected signal will be in general independent of the object depth. From 


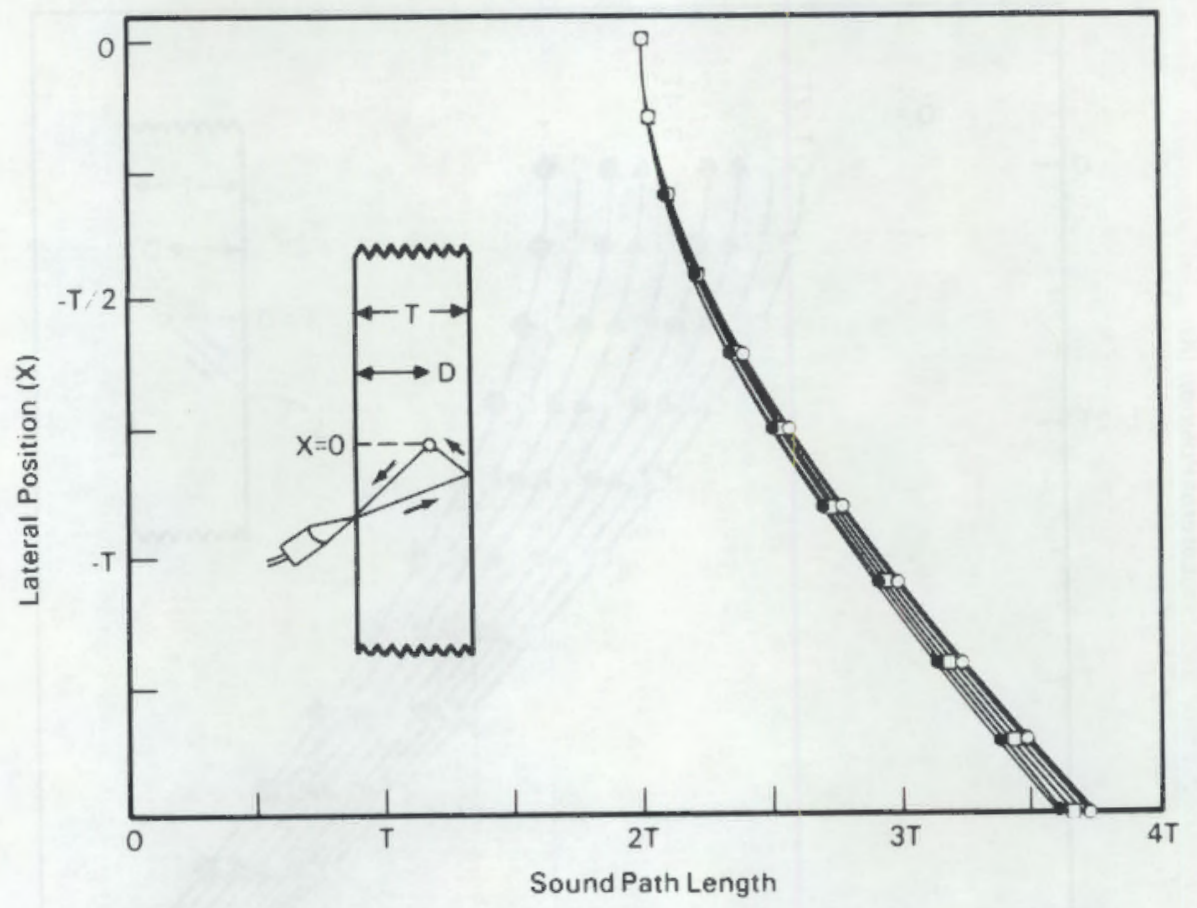

FIGURE 3. Curves Representing the Range for the P1-P3-P2 Sound Path in the SAFT-UT Pulse-Echo Mode

this elementary analysis, it appears that this bounce path would be difficult to incorporate into a SAFT imaging system since the expected propagation data is weakly indicative of the reflector depth. This fact is unfortunate, since this path is commonly encountered in normal ultrasonic data gathered with angle-beam illumination. This is especially true for vertically oriented planar defects. This phenomenon is the cause for the high amplitude 'corner trap' signal that is encountered when scanning this class of defects. It aids in the detection of these defects, but hinders accurately sizing the vertical extent of a given reflector.

Figure 4 shows a SAFT-UT image of a machined vertical sawcut in a stainless steel coupon. The data was collected in the SAFT-UT pulse-echo configuration. The notch, which simulates a vertical defect, was 0.6 inches in length and 0.3 inches deep into the material, and the coupon thickness was 0.585 inches. The image displays the B-scan side view and B-scan end view of the volume inspected. A tip signal has been imaged successfully in this example and the very strong corner reflection is apparent. As predicted, then, it would be difficult to size this defect in the vertical direction if the tip signal was not present. Also one may judge that this image represents a single continuous defect because of the duality nature of the indications, or he may judge that two independent reflectors on the same vertical plane are present 


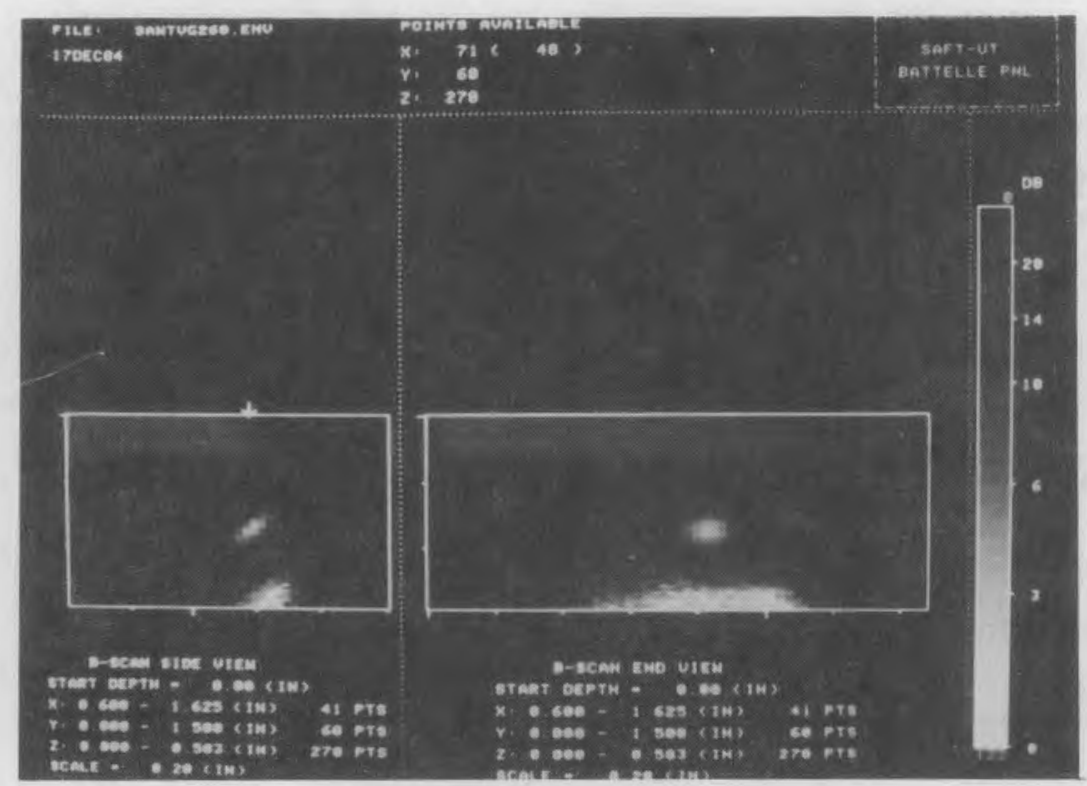

FIGURE 4. B-scan Side-View and B-scan End-View SAFT-UT Pulse-Echo Image of of a Vertically oriented Specular Sawcut in a Stainless Steel Coupon

in the material. Not enough information is available in the data to distinguish between these two cases.

For vertically oriented defects in materials with a finite thickness, the strength of the pulse-echo configuration lies in detection of these defects. The corner-trap echo is typically very strong and is present even in very attenuative materials.

\subsection{TSAFT MODE}

Tandem SAFT or TSAFT was implemented to provide a method for characterizing and sizing vertically oriented planar defects in materials with a finite thickness. The TSAFT configuration capitalizes on the forward scatter from the object rather than the direct back scatter as in the pulse-echo case. It consists fundamentally of a fixed transmitter that is placed in line with, or in tandem with, a scanned receiving transducer. The transmitter is placed such that the divergent sound beam illuminates the primary object area. The scanned receiver is then translated to receive direct energy reflected from the defect area. As in the pulse-echo configuration, after each pass of the receiver transducer, the transport mechanism is incremented so that a rectilinear pattern is obtained (e.g., around the circumference of a pipe).

To process the image of a TSAFT data file, four different paths may be considered as shown in Figure 5. To calculate these sound paths the part thickness 


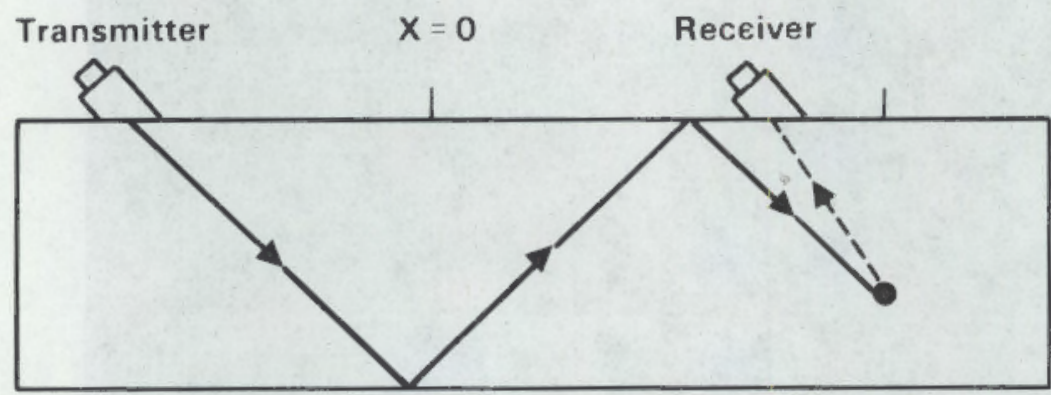

Path 1

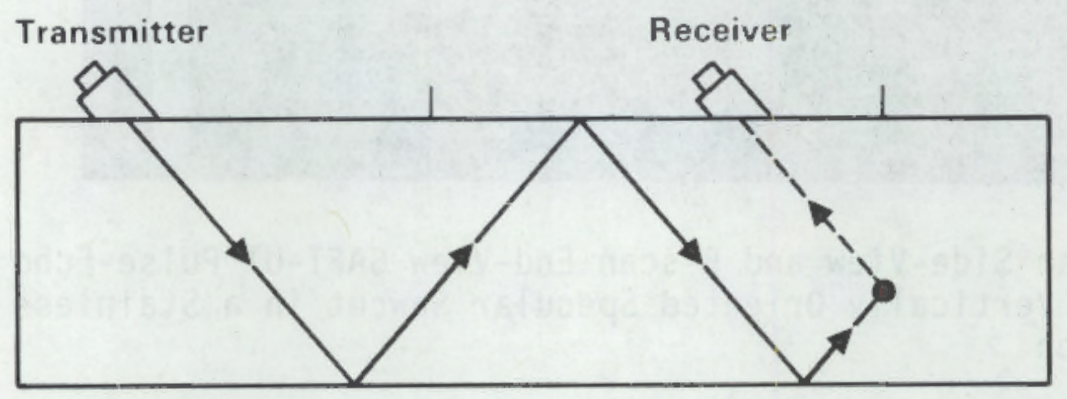

Path 2

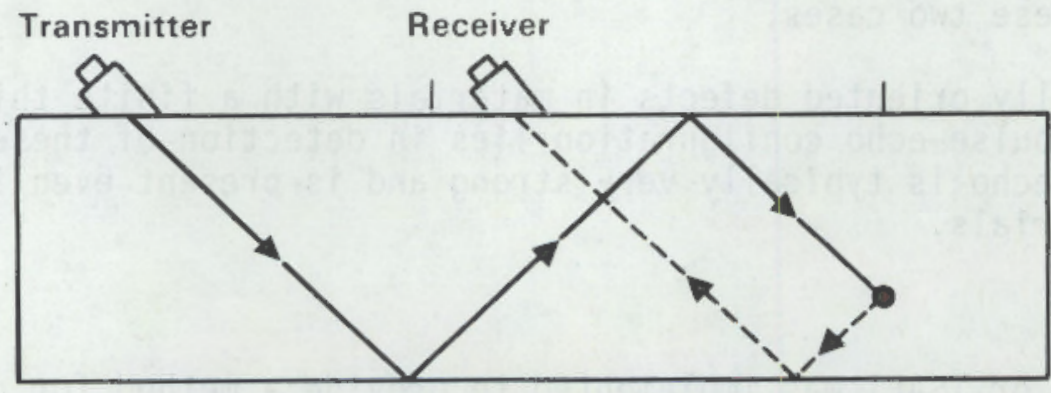

Path 3

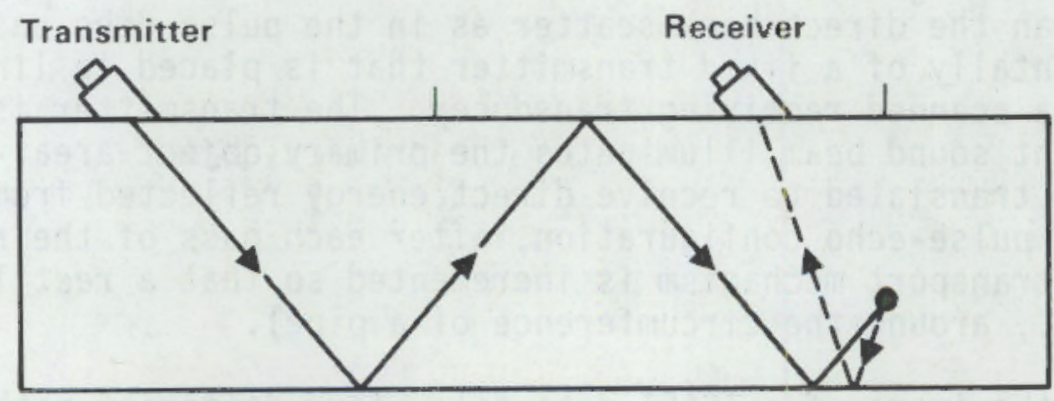

Path 4

FIGURE 5. Four Propagation Paths for Ultrasonic Energy Produced by the Tandem Transmission Technique 
and location of the transmitting transducer with respect to the scanned receiving transducer must be well known. Close analysis of Figure 5 shows that there are actually two transmit propagation paths and two receive propagation paths.

The transmit propagation paths are shown more clearly in Figure $6 . X_{T}$ and $X_{p}$ represent the $X$ coordinates of the transmitter and processing (object) point within a specimen of thickness $T$. To process the image point at depth D, the two corresponding transmit path lengths may be represented by:

$$
\begin{aligned}
& T_{A}=\left[\left(X_{P}-X_{T}\right)^{2}+(N T+T-D)^{2}\right]^{1 / 2} \\
& T_{B}=\left[\left(X_{P}-X_{T}\right)^{2}+(N T+D)^{2}\right]^{1 / 2}
\end{aligned}
$$

where $T_{A}$ and $T_{B}$ represent the path lengths corresponding to paths $A$ and $B$ respectively; and $\mathrm{N}$ is the number of times the ray encounters a reflective surface prior to striking the object point. In this example $N$ for path $A$ is 2 and $\mathrm{N}$ for path $\mathrm{B}$ is 3 .

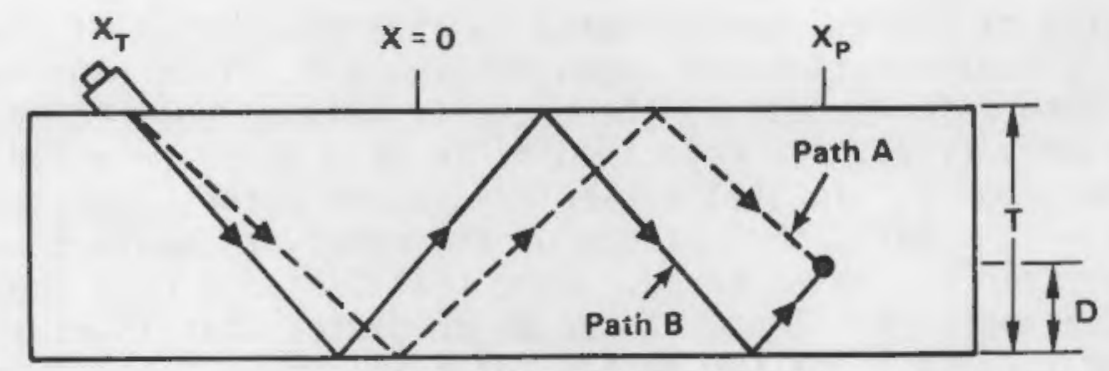

FIGURE. 6. Two Propagation Paths ( $A$ and $B$ ) from the Transmitter to a Point at $D$

Figure 7 shows the receive propagation paths to be considered. Here $X_{R}$ and $X_{p}$ represent the $X$ coordinates of the receiver and processing (object) point respectively. The resulting receive equations then become:

$$
\begin{aligned}
& R_{A}=\left[\left(X_{P}-X_{R}\right)^{2}+(T-D)^{2}\right]^{1 / 2} \\
& R_{B}=\left[\left(X_{P}-x_{R}\right)^{2}+(T+D)^{2}\right]^{1 / 2}
\end{aligned}
$$




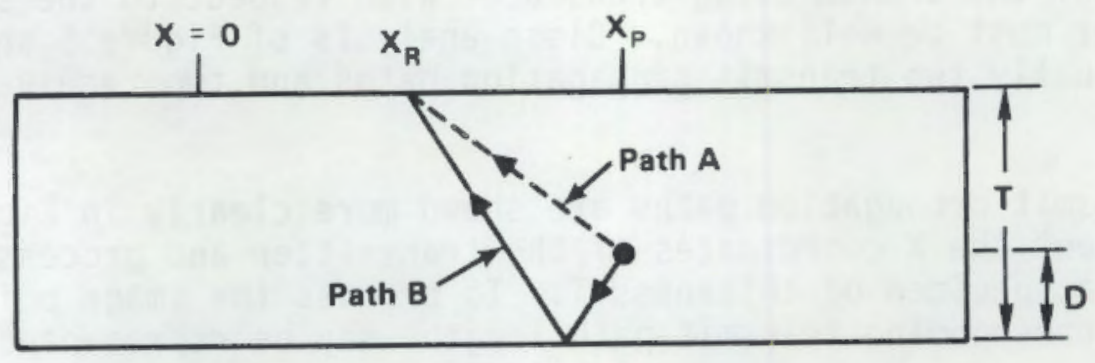

FIGURE 7. Two Propagation Paths ( $A$ and $B$ ) from a Point at $D$ to the Receiver

To determine the total sound path one needs to combine the appropriate transmit and receive equations. For example if Path 2 shown in Figure 5 is the dominant path, then the total propagation path is simply given by $\left(\mathrm{T}_{\beta}+\right.$ $\left.R_{A}\right)$. Typically in practice paths 2 and 3 shown in Figure 5 are established as the dominant paths for vertically oriented defects. Paths 1 and 4 may be more useful in specialized geometries.

The family of curves corresponding to the computation of the results of a TSAFT Path 2 configuration are shown in Figure 8 . This plot represents the total sound path with respect to the receiver lateral position for the center receiver ray only (i.e., the object point is held fixed relative to the receiver for each curve shown). In SAFT vocabulary, these curves represent the center A-scans only. This holds the effects of the receiver constant so the effects of the transmitter may be observed. Note that for each case there is uniqueness throughout the aperture. Thus, it can be predicted that TSAFT should provide an image that will describe the object fully in the vertical direction.

Figure 9 shows the tandem SAFT image of the identical sawcut described earlier with the pulse-echo case. Notice that, as predicted, the full extent of the surface of the vertical object is represented. Using TSAFT in this example provides the information to the interpreter so that a judgement may be made concerning the vertical extent of the defect. The image is independent of the tip diffracted signal. Also it is plain to the observer that there is a single vertical defect and not two co-planar defects.

Processing the TSAFT data file in the Path 2 mode produces interesting results if the data is acquired properly. The user may select to continue processing "beyond the back surface" (i.e. extend the image space to twice the thickness of the part), and the resulting image will contain the mirror or conjugate image appearing below the back surface. This should not be too surprising since the far surface acts like an acoustic mirror. The mirror image is actually the Path 3 image projected below the far surface. This image can be used, particularly in sizing edge connected defects using TSAFT2, and will be discussed in the subsequent section. 


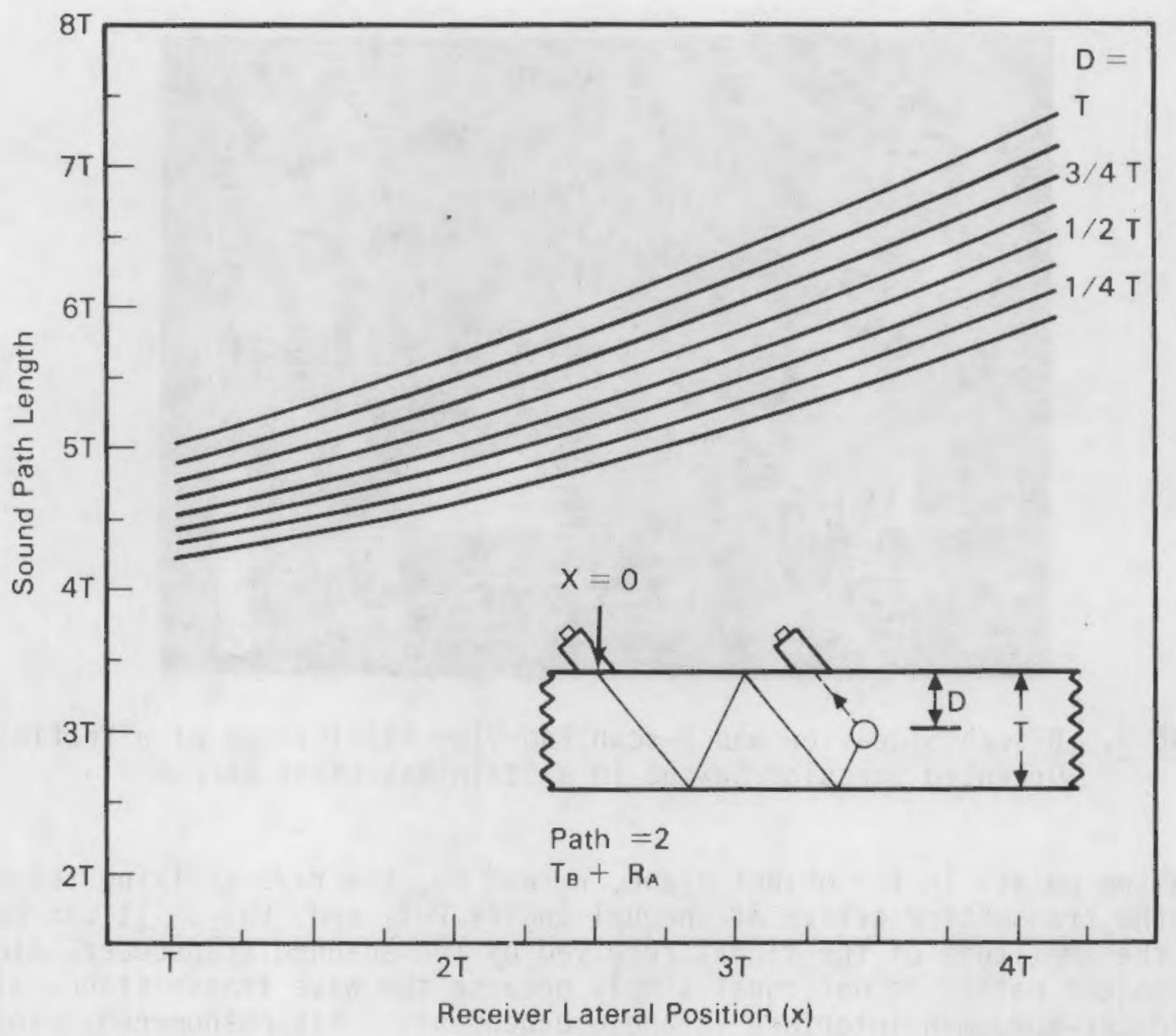

FIGURE 8. Path 2 Curves of the Total Center Ray Transmitter Sound Path for a Point Reflector at Depth D

\subsection{TSAFT-2 MODE}

When considering inspection of certain materials, defects may exist at any depth in the specimen--from small cracks located at the far surface, to deep cracks or fabrication defects that will reflect energy near the scanned surface. A full vertical object plane needs to be uniformly illuminated in this type of specimen in order to accurately determine the nature and size of the defects. Analysis of the tandem SAFT configuration reveals that illumination throughout a vertical object plane is in general not fully uniform. This is due to the fact that the transmitter is stationary and, due to laws of diffraction [6], will exhibit a non-uniform intensity distribution (beam pattern) throughout the illumination aperture. This is easily seen by observing figure $10(a)$. 


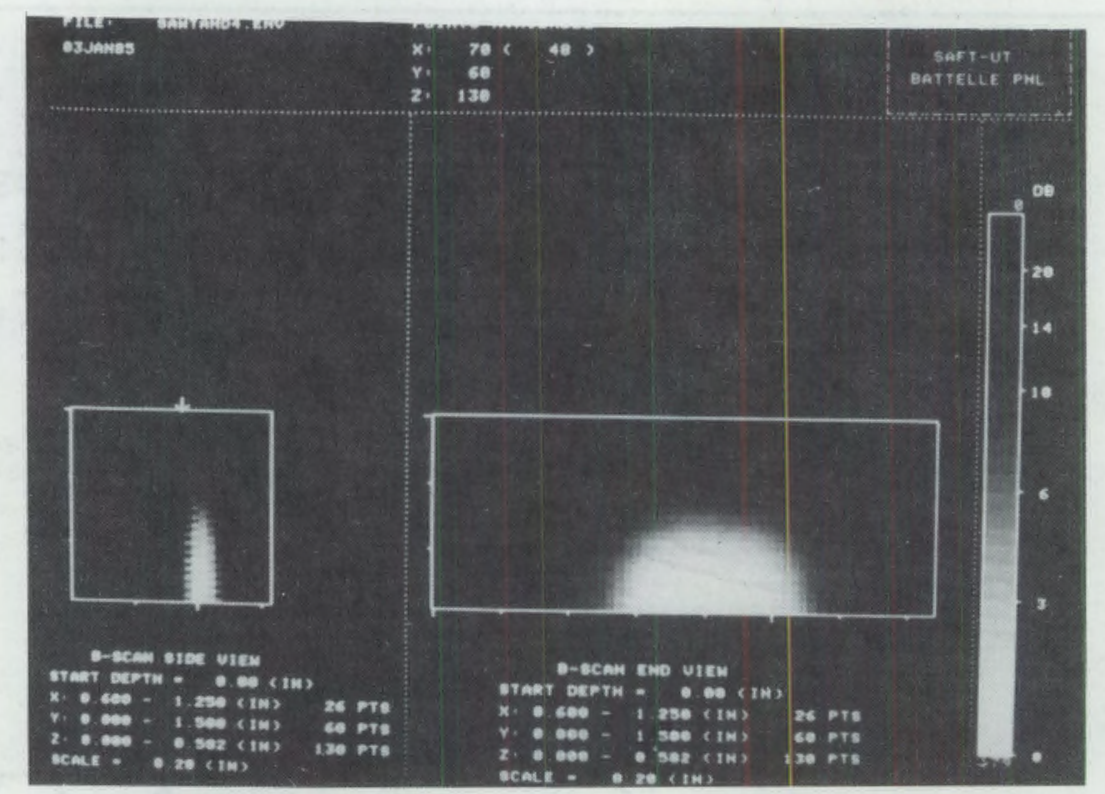

FIGURE 9. B-scan Side-View and B-scan End-View TSAFT Image of a Vertically Oriented Specular Sawcut in a Stainless Steel Coupon

Given two points in the object plane, $P_{1}$ and $P_{2}$, the rays striking these points from the transmitter arrive at unequal angles $\mathrm{PHI}_{1}$ and $\mathrm{PHI}_{2}$. It can be shown that the amplitude of the signal received by the scanned transducer, along independent paths, is not equal simply because the wave transmittance at the transducer-specimen interface is angle dependent. This phenomenon leads to undersizing when the object of interest extends beyond about half the beamwidth (insonification angle).

An alternative tandem technique (TSAFT-2) was implemented to alleviate the illumination deficiency of the TSAFT configuration. This second configuration may be seen in Figure 10 (b). To maintain a constant illumination throughout a given object plane, the transmit transducer is scanned at an equal rate as the receive transducer, but in exactly the opposite direction. For each vertical object plane, the angles $\mathrm{PHI}_{1}$ and $\mathrm{PHI}_{2}$ are equal, thus ensuring that the total center ray path length is constant, from the transmitter to receiver. This characteristic has proven to be valuable with full volume imaging; in particular it has enhanced the imaging of near-surface defects.

Figure 11 shows a comparison view of two SAFT-UT scans of the same object. Two opposing vertical sawcuts (semi-circular) were placed in a 1.0inch-thick aluminum coupon. They were machined such that there was a $0.050-$ inch separation at the center of the part. These objects were scanned implementing 5.0-MHz contact transducers in the 45-degree shear-mode configuration. The upper image shows the B-scan side view and B-scan end view of results obtained from the TSAFT configuration (fixed transmitter). It can be easily 


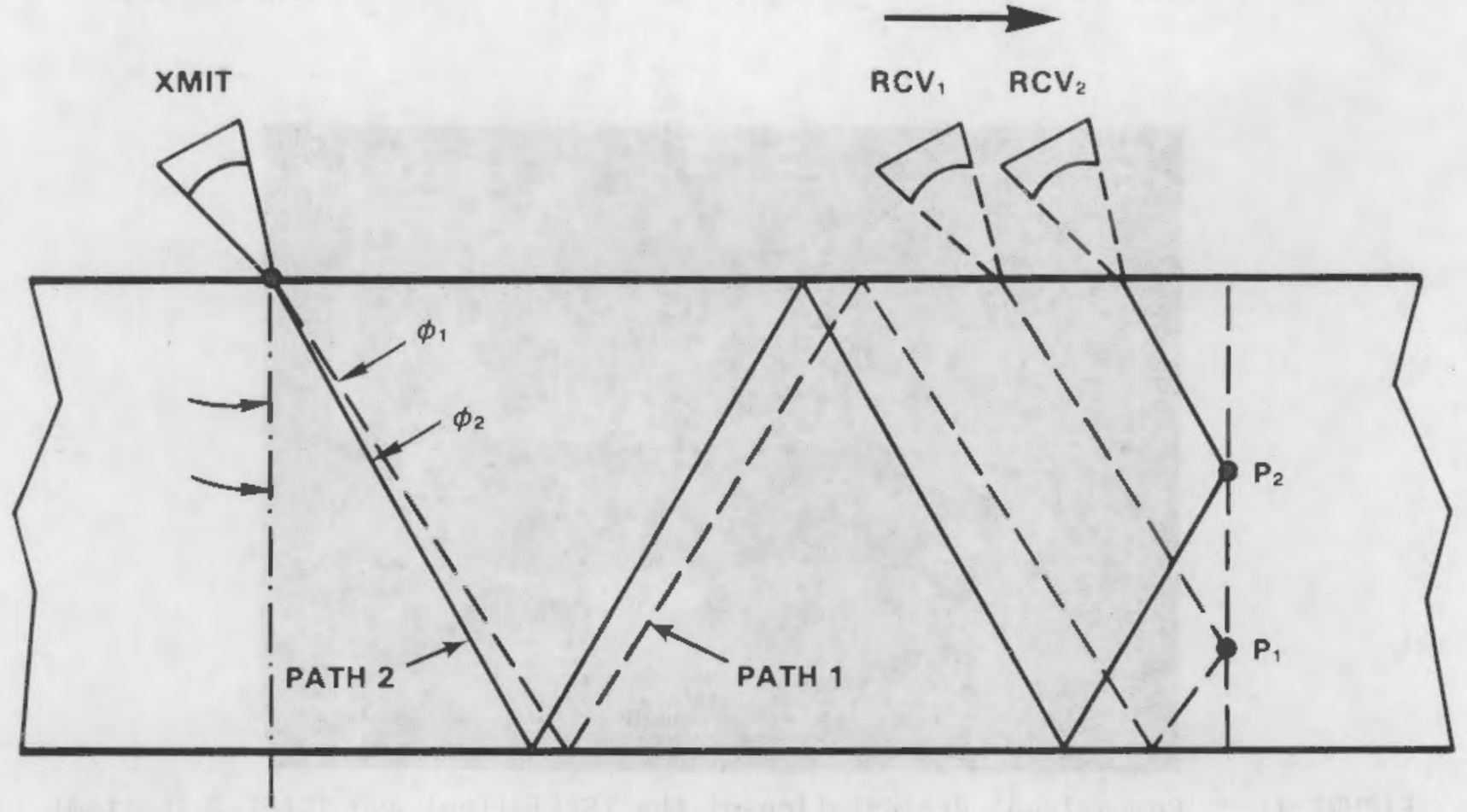

FIGURE $10(a)$. TSAFT Center Ray Paths for Point Reflectors $P_{1}$ and $P_{2}$

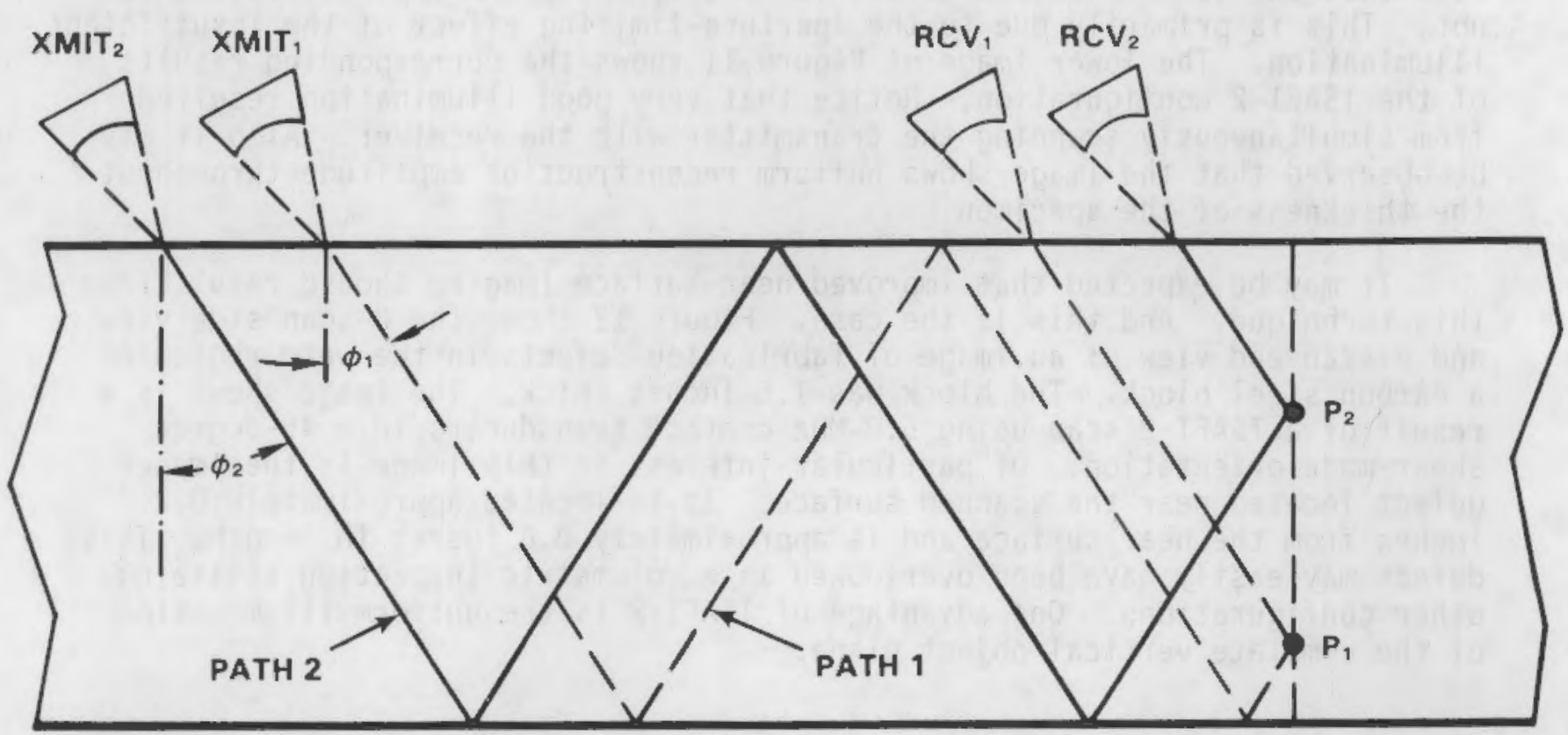

FIGURE $10(b)$. TSAFT-2 Center Ray Paths for Point Reflectors $P_{1}$ and $P_{2}$ 


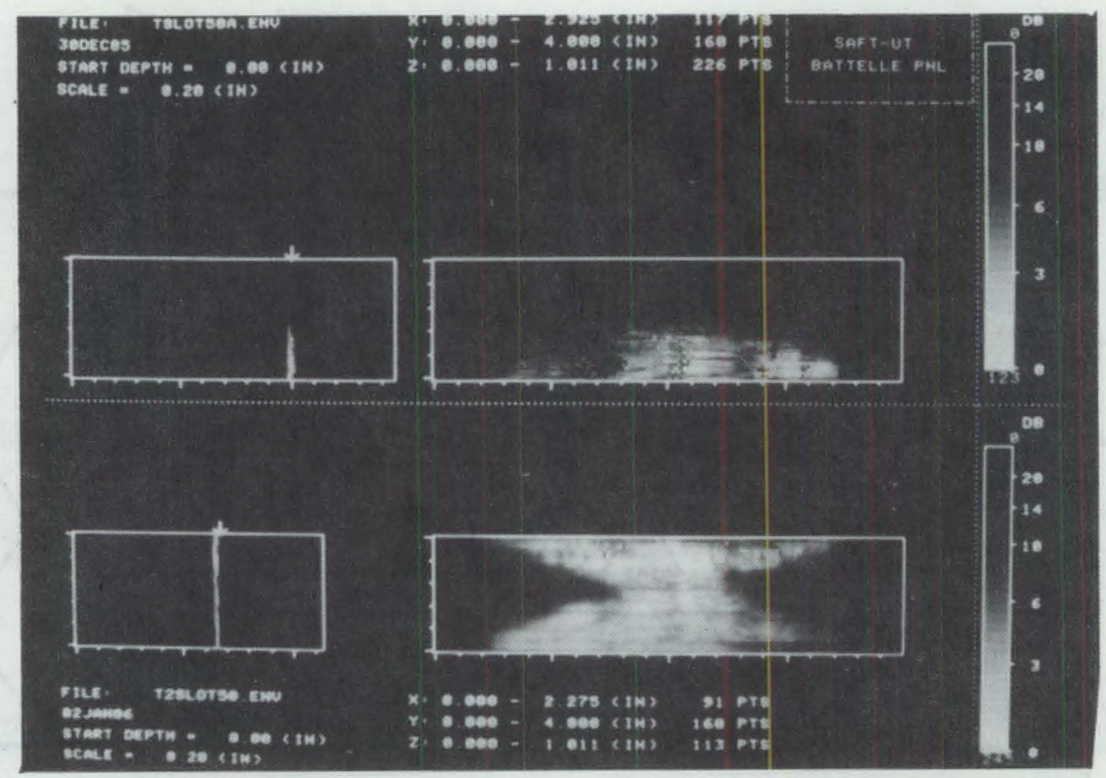

FIGURE 11. 'Comparison' Presentation of the TSAFT (top) and TSAFT-2 (bottom) Images of Two Opposing Vertical Sawcuts in an Aluminum Coupon

seen that the lower machined defect is imaged, while the upper structure is not. This is primarily due to the aperture-limiting effect of the insufficient illumination. The lower image of Figure 11 shows the corresponding results of the TSAFT-2 configuration. Notice that very good illumination resulted from simultaneously scanning the transmitter with the receiver. Also it may be observed that the image shows uniform reconstruction amplitude throughout the thickness of the specimen.

It may be expected that improved near-surface imaging should result from this technique. And this is the case. Figure 12 shows the B-scan side view and B-scan end view of an image of fabrication defects in the weld region of a carbon steel block. The block was 1.5-inches thick. The image shown is a result of a TSAFT-2 scan using 5.0-MHz contact transducers in a 45 -degree shear-mode orientation. Of particular interest in this image is the imaged defect located near the scanned surface. It is located approximately 0.2 inches from the near surface and is approximately 0.8 inches in length. This defect may easily have been overlooked in a volumetric inspection utilizing other configurations. One advantage of TSAFT-2 is the uniform illumination of the complete vertical object plane. 


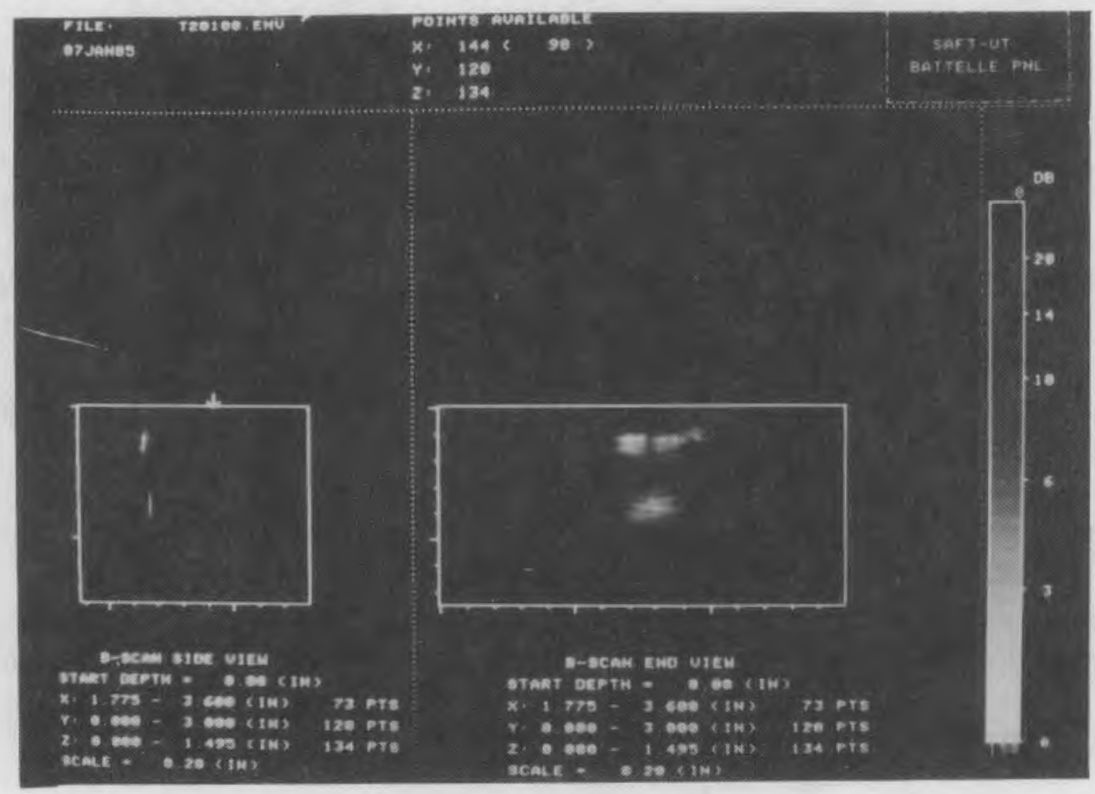

FIGURE 12. TSAFT-2 Image (B-scan Side-View and B-scan End-View) of Fabrication Defects in Carbon Steel Specịmen

\subsection{COMPUTER IMPLEMENTATION OF SAFT-UT}

Each data file acquired for SAFT processing consists of a series of data records preceded by a header area containing file parameters. These parameters communicate to the SAFT utilities associated information related to material properties, scan configuration, and processing parameters. The data records following the header area contain the complete digitized return signal for each location of the scan aperture. Each data record also contains a local header with information relating to the specific location and data of that record. So each data file consists of an orderly set of data and all information necessary to process that data.

The typical SAFT processing utility will first fetch the complete file into memory and begin operating on the data as a volume. A set of look-up tables are generated prior to processing that define relative addresses of each element to be summed for every specific location in the image space. These tables contain the results of computing the characteristic surfaces for each element in the records.

In the single transducer pulse-echo mode this surface has a hyperbolic shape as described earlier. Since the material is assumed to be homogeneous, and the scan is assumed to be orderly and rectilinear in shape, then each record has identically the same set of hyperbolas associated with it. Therefore, what needs to be precalculated and stored in the tables is a relative address with respect to the center processing element. 
Figure 13 graphically shows the computation method for the pulse-echo SAFT configuration. For each element in the data set, all off-center elements intersecting the characteristic hyperboloid and within the specified beamangle, are summed to. produce the final result for that element. Normally after processing is completed on all elements of a given record, envelope detection is performed to eliminate the carrier frequency component of the result. The next record is then selected for processing until the full resulting data set has been computed.

One can see from this description that the SAFT algorithm fundamentally consists of a great number of iterative fetch-and-sum operations. This operation occurs in what is many times referred to as the "inner loop", and becomes the focus when considering increasing the processing speed of a SAFT operation. 


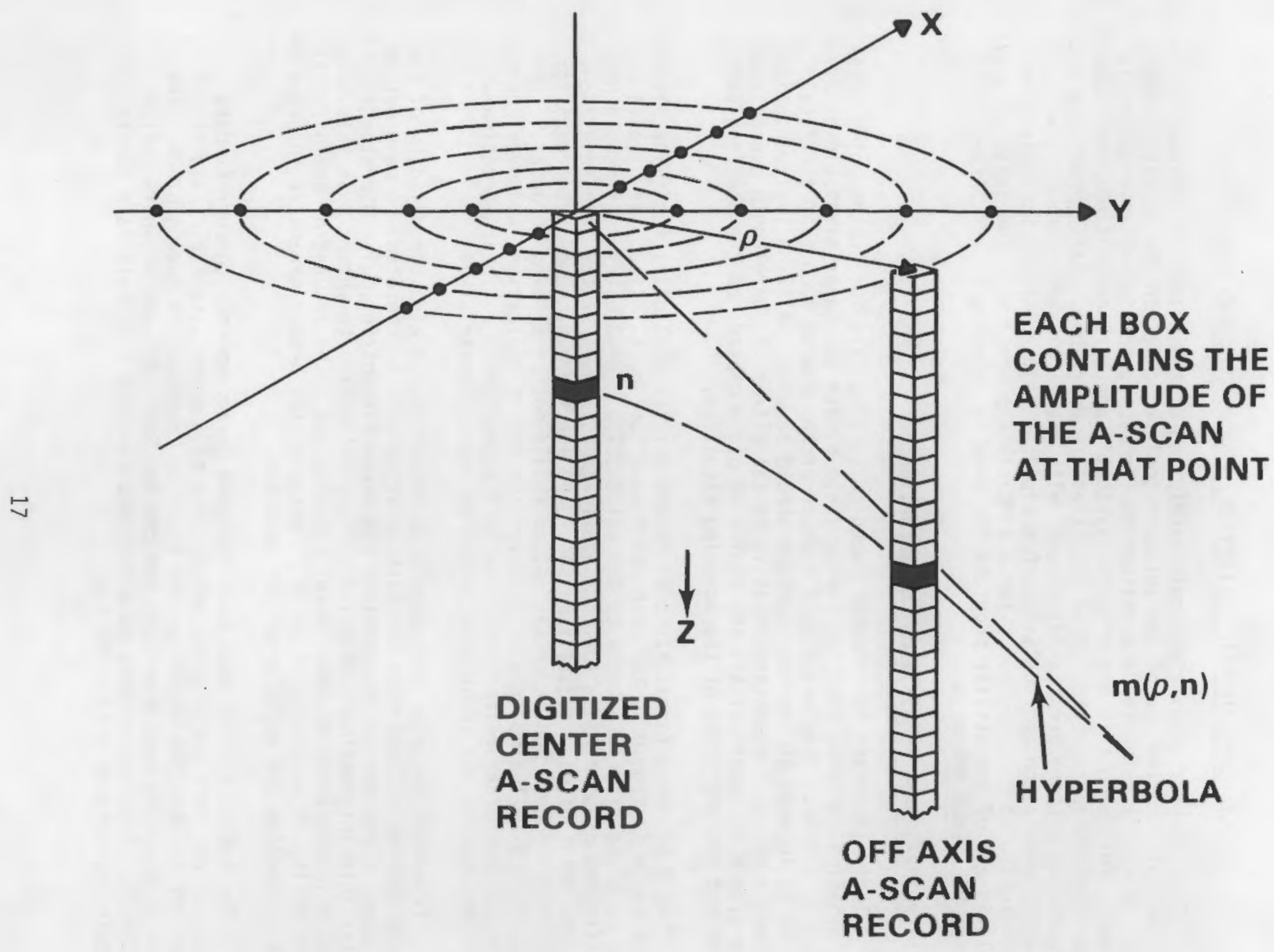

苛

FIGURE 13. Pictorial Representation of the SAFT-UT Coherent 


\subsection{SAFT-UT DATA COLLECTION, PROCESSING, AND ANALYSIS}

This chapter gives a general description of the practical implementation of SAFT-UT, and the use of the software tools supplied in the utility program set. First, a guide to data collection for subsequent SAFT-UT processing is given. Following this description, synthetic aperture generation is discussed, and miscellaneous preprocessing utilities are described. SAFT processing and associated options are then discussed, with the final section dealing with image presentation and analysis. Descriptions of the SAFT-UT utilities are highlighted in this section. For a complete explanation of the features available in all of the utility programs the user is referred to the SAFT-UT Utilities REFERENCE MANUAL.

\subsection{PRIMARY DATA ACQUISITION CONSIDERATIONS}

It is important to remember, when collecting data for subsequent processing by a SAFT algorithm, that the energy field within the material should be divergent in nature. This means that focused probes placed such that the focal point is located at the near surface should be used. Alternatively, contact probes with small diameters relative to the ultrasonic wavelength can be used. The effect is identical and the choice of probe depends on the specimen under test and the logistics of the scanning situation.

An 8-bit data format has been chosen for the SAFT utilities. The allowed data range is from 0 to 255 with the data offset by 127 (i.e., zero volts on the rf signal is represented by the value 127). The 8 -bit format should provide sufficient dynamic range for most applications. However the gain adjustment at the point of data acquisition should be set for maximum dynamic range for the object echo. Nonlinear saturation should not be allowed to occur if one expects the SAFT algorithm to discriminate object indications and overlapping signals from other reflectors. The SAFT algorithm is a linear operation and as such expects the input data to be reasonably linear also.

Care must be taken to ensure data integrity and also the validity of the parameters associated with the particular data file generated. A menu driven program is recommended to complete the parameter entries. The operator should enter this information just prior to data collection to ensure that this information is completed at the time of scanning and to be assured of the greatest probability of accuracy. A delay in entry of the header parameter information may jeopardize the validity of the data-set.

The SAFT utilities have been designed to accommodate concurrent data acquisition, SAFT processing, and display of a given data-set. This allows the user to view the result as the scanning operation is taking place. The data acquisition module written for the NRC SAFT implementation is called SAFTMAIN. This may be used as a template to adapt alternate data acquisition modules to achieve this operation. 
Concurrent data collection, processing and display is accomplished by utilizing shared data files, intermediate communication files, and synchronization with VMS event flags. This has been shown graphically in Figure 14. The data acquisition module, which usually is user dependent, generates the unprocessed data file as it scans. This module spawns the SAFT processing module as a child process and flags it each time a new scan line is available in the data file. The SAFT processing module reads and processes each available scan line of data, then it subsequently places the result in the processed data

Software Modules

Related Files

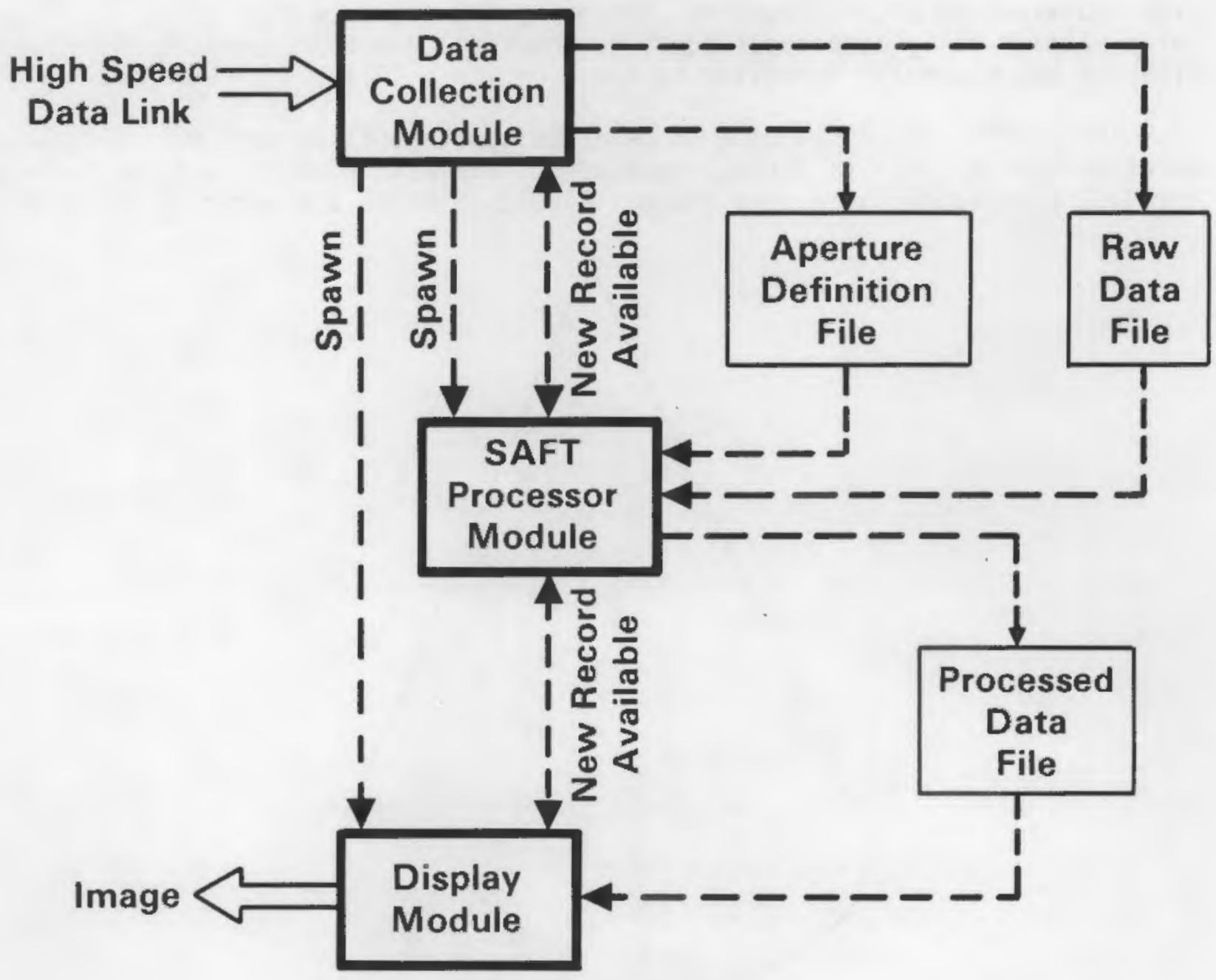

FIGURE 14. SAFT-UT Host Computer Software Configuration 
file. The SAFT processing module in turn flags the graphics module, which is also a child process, each time a new line of processed data is available. The result is an image being built and displayed as the processing is being performed.

The speed of this operation, of course, is limited to the slowest link in the chain. With the Real-Time SAFT Processor performing the processing module calculations, real-time rates are achievable.

\subsection{APERTURE DEFINITION}

The synthetic aperture definition chosen for processing is normally based on the beam-angle specified in the header of the specific data file. This angle determines the extent of the aperture cone, and thus, affects the image resolution and expected processing time. Normaliy the aperture definition is calculated automatically at processing time, however a pre-made aperture definition file may be optionally specified by the user.

The GENAPT utility is used to generate aperture files that may be selected to cause special effects during processing. The most common aperture shape used for SAFT processing has a cone shape. Figure 15 shows a diagram of this typical

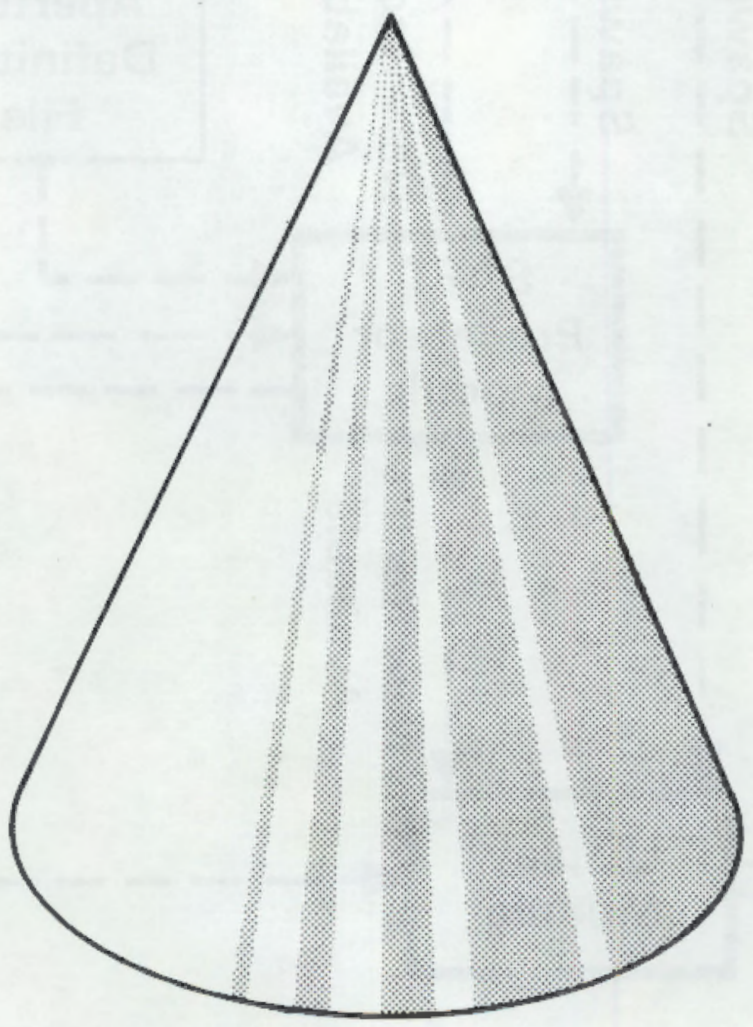

FIGURE 15. Normal Beam 3-Dimensional Synthetic Aperture Geometry 
aperture. While processing a given record, all datum that lie within this shape relative to this "center A-scan" are summed to form the resultant Ascan. If sampling began at the front surface of the material, then the cone comes to a value of unity at the near surface and expands at a constant angle as the beam penetrates the material. If data sampling began below the near surface of the part, as happens many times in thick materials, then the aperture shape is a conical section beginning at that depth in the material. One of the options that may be chosen in the GENAPT utility is limiting (clip radius) the extent of the aperture cone. This may be necessary to keep files of very thick specimens from generating an unreasonable large aperture cone.

The user may also choose to select line-SAFT processing by simply adjusting the aperture shape. This is done by selecting the LINE command. Normally line-SAFT processing is performed along the scan (X) axis, but $Y$-axis lineSAFT is also available. In X-axis line-SAFT the aperture is defined such that no off-center points are summed from records lying outside of the current $X Z$ plane. In other words the 3-dimensional cone is collapsed into a 2dimensional triangle.

The approximation of having the aperture cone come to a point at the near surface has proven to be inaccurate when the diameter of the transducer becomes significant. The user may select a "beam entry diameter" that more accurately represents the effective diameter of the actual sound field at the near surface. This value is set in the data file header block using the DEDIT utility. If this value is non-zero then GENAPT will generate a cone that is shaped similar to a funnel as shown in Figure 16, with a beam entry diameter $D$. The number of off-center A-scans that will be summed near the surface of the part, then is dependent on the value of the "beam entry diameter".

More exotic aperture shapes are also available. The TROUGH command generates an aperture cone definition with the center dug out of it. The cone shape is as if a shovel was moved along the scan $(X)$ axis to scoop out the center of the cone. This is shown in Figure 17. This shape may be chosen to process only the high spatial frequencies in the $Y$ direction. It may prove to be helpful for distinguishing between long specular reflectors (geometric reflectors) and diffuse reflectors (irregular defects). The width of the trough is defined by the "beam entry diameter" parameter located in the header area of the data file.

Another specialized shape provided in the GENAPT utility uses the DOUGHNUT command. This command generates a doughnut shaped aperture cone definition as shown in Figure 18. The center elements near the fundamental element then are not summed during processing when this geometry is chosen. This option may be chosen to specify processing that will not include any low spatial frequencies in its computation. The diameter of the hole in the middle is again defined by the "beam entry diameter" header parameter of the data file. 


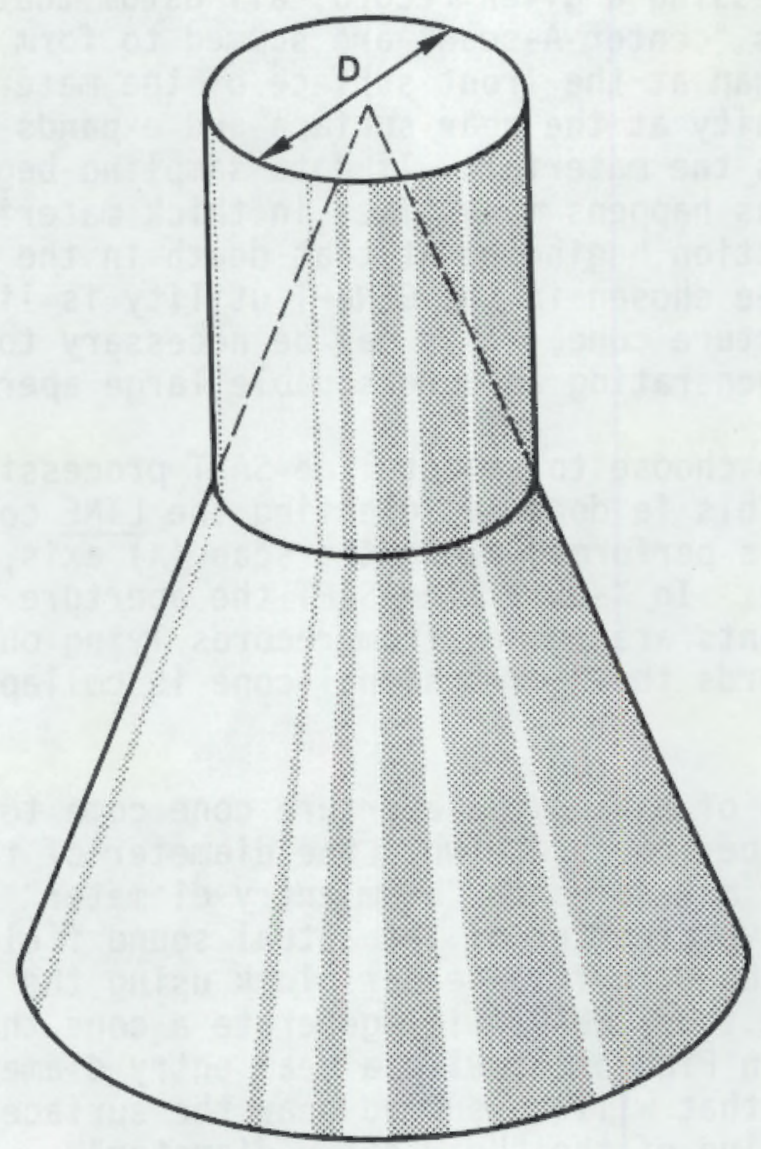

FIGURE 16. Normal Beam 3-Dimensional Synthetic Aperture Geometry with a Finite "Entry Point Diameter" (D) Specified

The DUMPAPT utility provides a way of viewing the contents of a generated aperture definition file. It displays the contents of the "limit list" which specifies the number of elements that are to be summed for each element ( $z$ value) in the A-scan records. Two other significant arrays are displayed that define the relative $X$ and $Y$ indices for each of the elements to be summed. These are displayed in the order of summation.

\subsection{PREPROCESSING TOOLS}

The SAFT-UT data file editor, DEDIT, is provided as a utility to edit any portion of a file in the SAFT-UT data file format. Individual data points and records may be edited, but the primary purpose of this utility is to allow interactive editing of the data file parameter header information. All relevant header values may be modified with this utility. 


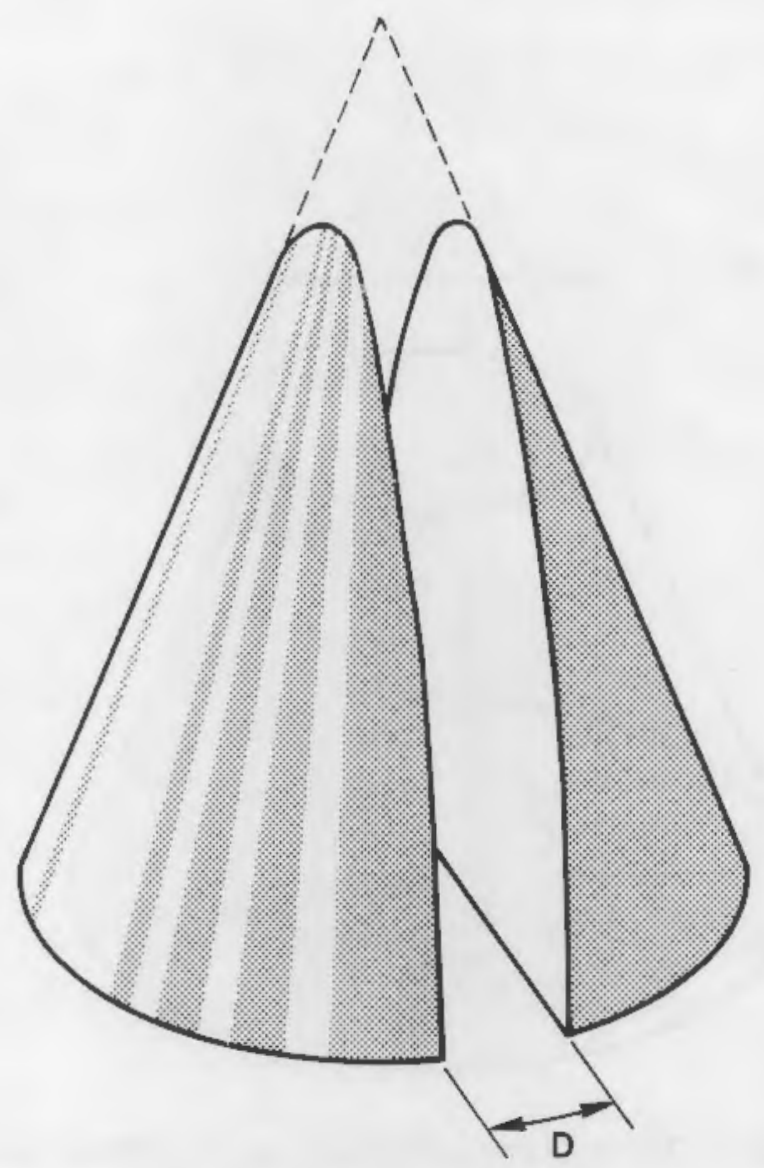

FIGURE 17. Normal Beam 3-Dimensional Synthetic Aperture Geometry, with the THROUGH Option Selected and an "Entry Point Diameter" of D

The data set statistics (minimum, maximum and average) may not have been calculated by the particular data acquisition system. The CSTAT utility will calculate the statistics of the data set and place the result in the data file header. Also this program calculates and updates the individual record statistics.

For wide-band applications a time derivative of the data set is necessary for optimum image quality (best resolution). The DFDT utility performs this operation. No effort was made to optimize the computational efficiency of this utility, so ten to fifteen minutes may be required to perform this operation.

The DFTRIM utility allows the user to trim any axes $(X, Y, Z)$ of a given data file. Thus an unwanted signal may be removed, or more likely a very large data file may be reduced in size to facilitate file management. 


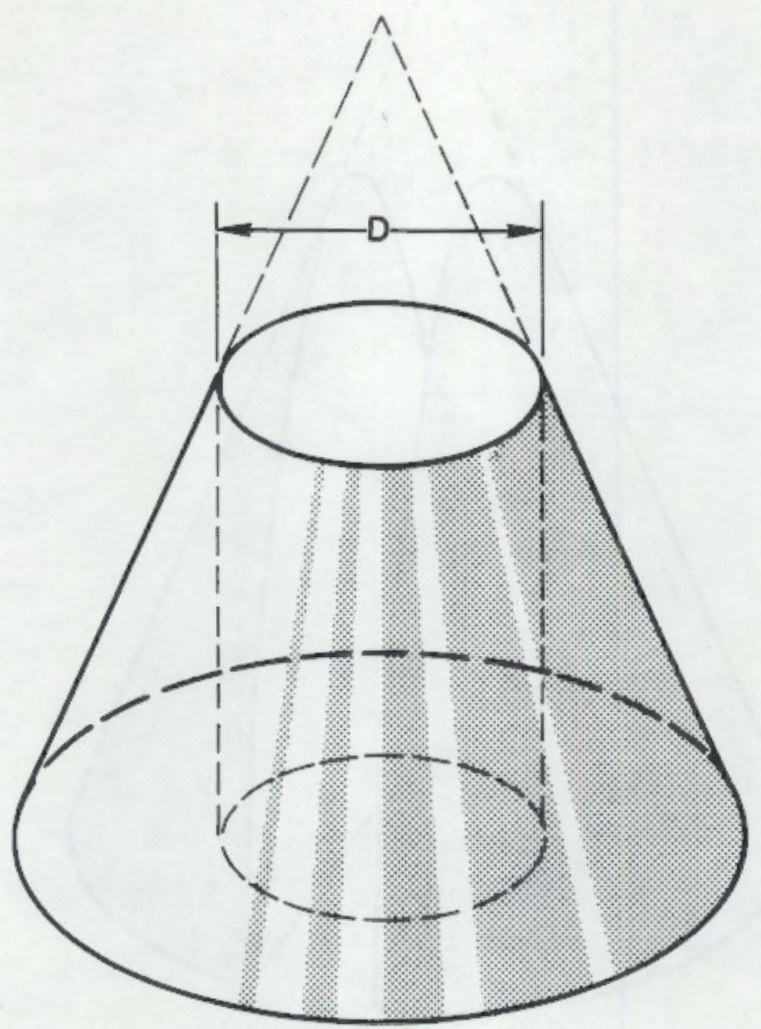

FIGURE 18. Normal Beam 3-Dimensional Synthetic Aperture Geometry, with the DOUGHNUT Option Selected and an "Entry Point Diameter" of D

A filter program has been supplied (FILTER) to assist in removing spectral information that may contribute only to the noise content of the image. This filter routine simply truncates frequencies outside of the selected range. No attempt has been made to generate a Gaussian distribution.

Three utilities have been supplied to modify the axes order in a data file. SHUFFLEX flips both the $X$ and $Y$ axes. This is useful if the incident angle is positive and is desired to be negative, or visa-versa. SHUFX flips only the $X$ axis. In effect both SHUFFLEX and SHUFX negate the incident angle sign. A negative incident angle is most common in SAFT data collection (see the section 6.0 which refers to the SAFT-UT Scanning Conventions).

SWAPXY will swap the $X$ axis for the $Y$ and the $Y$ axis for the $X$ axis. This is equivalent to rotating the axes by 90 degrees. This is helpful if the incident angle was originally in the increment ( $Y$ ) direction. By convention the SAFT utilities expect the incident angle to be in the direction of the $\operatorname{scan}(X)$ axis. 
The Fourier transform of a given file may be generated with the utility SPECTRUM. This may be used with more advanced techniques for signal processing and anaTysis in the frequency domain.

\subsection{PROCESSING THE DATA FILE}

The primary utility oriented toward performing SAFT processing is SAFTPROC. This utility may be invoked by simply entering 'SAFT' during an interactive session. It is a command driven utility that assumes a variety of defaults. After an input data file has been specified the user may type the command PARA to view the current defaults. Any or all of these parameters may be changed to suit the particular situation. If a user is content with the current parameters then the GO command will spawn a sub-process to execute the appropriate processing module corresponding to the parameters chosen.

A few techniques developed to improve the speed of SAFT processing have been implemented in the SAFTPROC utility. Selective processing will be discussed first. The selective processing method was developed through analysis of the SAFT-UT pulse-echo algorithm. If echoes are analyzed that would be expected from deep section material, it is noted that the data-sets generally are much larger than thin section data-sets, and the apertures are much larger since the divergent ultrasonic cone is broader. One other characteristic of this type of data-set is that often the observed background noise is less since the return echo is typically well separated from the large front surface signal. There tends to be a vast quantity of data points that are very low in intensity. Selective processing was devised to introduce some logic with respect to signal amplitude in order to minimize the quantity of inner loop operations necessary to process the data-set.

The SAFT algorithm may be thought of as a hyperbolic spatial matched filter. It can be seen that, for every elementary point object that provides an echo sufficient to be recorded, a hyperboloid is drawn that is weighted in amplitude. It has a high amplitude center point with the amplitude trailing off in lateral directions. The amplitude distribution around the center point should be predictable if the amplitude of that point is known.

If a single center A-scan element in the SAFT algorithm is at the location of the object point, then the amplitude at that center A-scan element will be larger than any of its corresponding locus of off-center A-scans. And conversely, the observation can be stated that if the given center A-scan element is not at the location of the object point, then its amplitude value will be much less than the element that is at the location of the object. So SAFT-UT selective processing makes the assumption that, within the SAFT summation iteration loop, if the amplitude of the center A-scan value is very low, then it is not at an object location and there is no need to sum the off-center values to obtain its final value. If the point has this condition, then its own amplitude is retained (or optionally zero may be chosen) for the final result, since it is assumed the off-center values will not contribute. 
So a threshold (clip or selective processing) level may be selected by the user. This value is entered in DB relative to the maximum data value in the data file. A value of $-20 \mathrm{~dB}$ is set as the default selective processing value. This corresponds to $10 \%$ of the maximum value. So, effectively, if the amplitude of the elementary data element being processed is below this threshold value, then no off-center elements will be summed. The action that occurs at this point is selectable. The raw data value may be entered, or zero may be entered. Experience has shown that this matters very little. If selective processing is undesirable, then simply enter $-50 \mathrm{~dB}$ or smaller and no clipping will result. The acceleration experienced in the SAFT processing calculations varies greatly from data file to data file. The greatest improvement is observed on thick material with little backscatter (noise). other techniques oriented toward expediting the processing of SAFT files are related to envelope detection and reducing data handling.

The result of SAFT processing contains a high frequency component (carrier frequency) that usually is eliminated prior to display. This operation is called envelope detection and is normally performed automatically in the SAFTPROC utility by selecting envelope detection $O N$. This detection scheme utilizes a convolution (sliding window) technique to describe the envelope.

If envelope detection was selected to not be performed during SAFT processing there are some stand-alone utilities that may be used to perform this as a subsequent operation. ENVELOPE will perform the convolution technique identical to that which may be selected during processing. Another utility called ENVDET will perform a Hilbert transform operation that results in a more exact envelope, but which generally takes much longer to execute. Also RECTIFY is available to simply perform the absolute value function on the data file. In addition to operating on the undetected processed data, these utilities may be used to prepare the unprocessed (raw) data file for display. Many times observing the unprocessed data helps in the analysis and interpretation phase of SAFT imaging.

The result of the envelope detection operation is a slow varying demodulated function compared to the original rf data-set. The requirements, therefore, on the temporal sampling are somewhat relaxed and one would expect that fewer data points are necessary to accurately describe the envelope detected signal. The sampling adjustments option in the SAFTPROC program, therefore, was initially devised to reduce excess data handling in the graphics modules.

The automatic sampling adjustment (A-scan record compression) will reduce the number of elements in each record by a factor based on the lateral $(X)$ increment. The depth ( $Z$ dimension) of the output pixel generated will be expanded to about half the size of the $X$ dimension. The element's $Z$ dimension in the input file is normally about 10 to 20 times smaller than the lateral increment size. Thus, a reduction of the output data file size will be from a factor of 5 to 10 with essentially no reduction in image quality. Automatic 
sampling adjustment will not effect processing time, but will improve system response due to the reduction of data handling.

The user may manually choose other sampling adjustments. There are four factors mentioned in the SAFT-UT Utilities REFERENCE MANUAL. The first two effect the lateral $(X, Y)$ sampling during processing, and are multiplication factors. Entry of 1 does no alteration. If 2 is entered for the $X$ factor, then every other record in the $X$ direction will be used for a center A-scan. The actual summation process, however, will utilize the entire data set. Thus the output file will have exactly half as much data as the input file, and processing will take half as long. Correspondingly the $Y$ factor operates similarly.

The last two manual sampling adjustment factors relate to temporal sampling. The $Z_{P R O C}$ factor defines a multiplication factor that specifies the sampling to be performed during SAFT processing. This is similar to the $X$ factor. For example if 2 is entered for this parameter, then every other point in the A-scan records will be processed. And, as with the $X$ factor, the actual summation of each processed point utilizes the entire data set. The $Z_{\text {ENV }}$ factor, however does not effect the processing whatsoever. It performs sampling adjustments on the $Z$ axis of the envelope detected output record. This factor is the parameter that Automatic Sampling Adjustment alters when it is selected.

\subsection{DISPLAY AND ANALYSIS OF THE FINAL IMAGE}

A number of display utilities are included to offer a versatile presentation of the processed images. Most of the development effort has been oriented toward display on a Ramtek 9465 graphics device. However one utility, TPLOT, has been supplied to operate with a Tektronix 4105 compatible graphics termina?.

TPLOT has been written to provide an alternate display device to the Ramtek image processor. Thus if another user is displaying an image on the Ramtek monitor, then one may be able to review a second image at the same time. Also in the case of a hardware failure TPLOT offers an alternative for temporary backup.

The user may display single views on the 4105 terminal with an eight color template. The views are limited to the B-scan side-view and the B-scan endview. C-scans (plan views) are not currently supported in TPLOT.

When viewing B-scan presentations it is important to remember the content of what is being displayed. The $Z$ axis usually contains an envelope detected signal. When a reflection occurs there will be a pulse of finite duration which is dependent on the bandwidth of the system. So a reflection from a surface may appear as a volumetric indication to the untrained eye because the pulse width becomes part of the B-scan display. Remember that the beginning of the pulse is the correct location of the reflector, not the maximum value 
of the pulse. This is important, especially, when viewing the B-scan sideview projection. It is proper then, to use the leading edge of the envelope for determining the size and the location of a reflector.

Graphics utilities available for the Ramtek 9465 device have been written to provide a versatile tool set for flaw detection, flaw sizing and image interpretation. A utility, CPLOT, has been included that quickly displays an image of the B-scan end-view and B-scan side view of the input file selected. This module may be used for concurrent display of an envelope detected file while processing of the file is still in progress. If the user is in a SAFTPROC interactive session, then entry of the CPLOT command will invoke this display module on the most recent SAFT processing (GO command) initiated during the current session. This serves as a handy preview of the results prior to completion of the SAFT processing.

Also a user may preview an envelope detected file being processed by another user. This is particularly useful if processing has been invoked on a batch queue and one wishes to evaluate the progress of its execution.

CPLOT will display the composite B-scan views of the input data file. If the file has a long $Y$ dimension relative to the $X$ dimension (as with weld inspection on piping), then the end-view will begin scrolling to make a sliding window of the most recent scans. A single slice side-view is displayed in the lower left-hand region of the screen, with the $Y$ location of this slice shown by a marker in the end-view. This gives the viewer a better knowledge of the content of the image, especially with complex reflectors.

The primary graphics toolbox developed specifically for analys is of SAFT files is the APLOT utility. It provides an extensive range of tools for viewing images and analyzing the characteristics of those images.

Upon entry of the APLOT utility the user is in normal 'plot' mode. A file is read into the session with the FILE command. If the PLOT command is

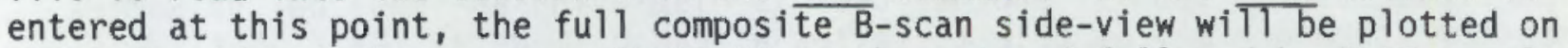
the left side of the screen. PLANE XY may be entered followed by PLOT to plot the B-scan end view on the right side of the screen. Or alternatively PLANE $X Y$ may have been selected to view the C-scan view of the image file. By default the two images displayed are scaled together (evenly scaled), and all dimensions $X, Y$ and $Z$ are scaled equally in both views. A selection of UNEVEN will free subsequent plotting to scale the individual views for maximum use of the available screen.

The user may choose to use the cursor to select an area of interest on the most recent view plotted. This is performed with the BOX command. A box is drawn around the area of interest and a subsequent PLOT command will expand the area of interest to view it in more detail. In this way a subset of the image may be easily viewed. Entry of the PARA command will inform the user about the current coordinates of the subset being displayed. Alternatives to 
the BOX command are the $\underline{X}, \underline{Y}$, and $\underline{Z}$ commands. These explicitly select subregions of the three axes. that:

Cursor operations are also available (always on the last view plotted)

1) allow the image to be nomalized by the value at the cursor location,

2) display the value and coordinates of the data point at the cursor location, and

3) display the distance between two cursor entries for feature sizing.

The 'compare' mode allows the user to simultaneously view images of two independent files. This is very useful when viewing files of different configurations (pulse-echo and TSAFT-2) representing the same physical volume. To enter the compare mode, select a file of interest with the FILE command, then enter COMPARE T. This will plot the B-scan views of this file in the upper portion of the screen. The second file is then retrieved with another FILE command. Entry of COMPARE B will then plot the corresponding views of this file on the lower portion of the screen. Cursor operations are not allowed in compare mode, but all other operations are valid.

The T-VIEW option is available if the user wishes to plot a view from the transducer's point of view. This operation only makes sense for angle beam files, since a C-scan is equivalent to a T-VIEW presentation for normal beam configurations. The T-VIEW presentation is as if the user is looking down the ends of the A-scans. That is, the display is a projection perpendicular to the angle of insonification. This mode is invoked on the FILE command line as described in the SAFT-UT Utilities REFERENCE MANUAL.

APLOT also allows plotting of individual A-scans. This may be particularly useful for observing waveforms in an unprocessed data file. This is accomplished with the ASCAN command. The number of points plotted may be windowed with the $Z$ or BOX command.

The spectrum of a selected waveform may also be plotted using the SPECTRUM command. The portion of the waveform used to compute the Fourier transform may be restricted using the $Z$ or BOX command as with the ASCAN command. This is helpful if nonlinear portions of the A-scan (such as front surface signals) are unwanted in the computation of the spectrum.

APLOT displays may be saved (written to a disk file) for future reference. This operation is accomplished using the SAVRAM command. The RESRAM command performs the retrieval. Both SAVRAM and RESRAM are also stand-alone utilities that may be invoked outside of an APLOT session. 


\subsection{SAMPLING AND RESOLUTION REQUIREMENTS}

A variety of performance evaluation studies have been performed to more accurately define SAFT-UT strengths and limitations, and to assist in forming operating procedures for inspections. Some of the results, related to system resolution and sampling criteria, will be stated here.

\subsection{SYSTEM RESOLUTION}

The quality of the final image is determined by the various components used in the system. There are basically four components that effect image quality. These are transducer characteristics, the aperture size chosen for SAFT processing, the physical scan aperture, and the display resolution.

The transducer characteristics are fundamental when describing image resolution. The first and most obvious parameter to consider is the transducer center frequency. This of course is inversely proportional to the wavelength of the sound in the material. The resolution of the system is nearly always directly proportional to the ultrasonic wavelength. So it is important to select a maximum operating frequency for the inspection. This must be balanced with signal-to-noise ratio, and also the digitizing sampling rate that is available on the data acquisition system.

SAFT also requires that a divergent sound field be generated in the material under inspection. The width of this cone is commonly referred to as the "beam angle" (a). The true beam angle of the transducer determines the image resolution capability of that particular transducer.

The bandwidth of the transducer and the associated electronics also effects the system resolution. The coherent summation algorithm is a narrow band approximation [5] that requires a temporal derivative operation in order to process wideband data files. A good explanation of bandwidth effects is given by Busse, Collins and Doctor [1]. Bandwidth contributes to both the depth and lateral resolution of an image. With a coherent summation system, as the bandwidth increases the depth resolution improves, but at the cost of the lateral resolution. So an optimum balance needs to be a used. PNL uses a bandwidth criterion based on plotting depth resolution verses bandwidth and lateral resolution verses bandwidth. The intersection of these curves produces a uniform volume resolution, and is considered optimal for most applications. A 50 to 60 percent bandwidth is typical for most SAFT-UT applications.

(a) In contrast the central ray of the transducer, measured relative to an axis perpendicular to the inspection surface is the associated "inspection angle." 
Prior to SAFT processing, a synthetic aperture beam angle is selected. This defines the extent of the synthetic aperture cone. Ideally, this angle is characteristic of the actual beam angle of the transducer. However, the synthetic aperture is a binary function; the off-center A-scans within the cone are summed, the off-center A-scans outside the cone are not summed. So it is a judgement decision on the user's part to choose the value of the synthetic aperture beam angle. As the synthetic aperture beam angle is increased the number of summations is increased; thus increasing the processing time. Also, because the final summation is averaged by the number of points summed, the dynamic range of the output image will be reduced by increasing the beam angle parameter. However, reduction of the synthetic aperture beam angle parameter directly reduces the sharpness or resolution of the resulting image. Generally, the angle corresponding to the $-6 \mathrm{~dB}$ level of the transducer beam profile is a practical value for most applications.

The physical scan aperture size effects the resolution only if it is not large enough to contain all of the required off-center A-scans when processing the object space. This is called "aperture limiting". Each image point is a summation of a locus of points. If the dimensions of the scan are such that not all of this locus is available, then the resolution of the image at this location is degraded. Because of this the resolution of all SAFT-UT images is significantly reduced along the edges of the resulting image. Care must be taken to ensure the object area is well within the scan boundaries so that all off-center A-scans will be available for processing the area of interest.

\subsection{SAMPLING CRITERIA}

Since the SAFT-UT algorithm consists of discrete digitized time domain waveforms, sampling effects need to be considered. There are two areas of sampling in this system: temporal sampling and lateral sampling.

For 3-dimensional SAFT operations (volumetric synthetic aperture cone) certain guide-lines have been determined. The lateral ( $X$ and $Y$ ) scan sampling should be at least two samples per resolution element size. So, for example, if a resolution of $0.1-i n c h e s$ is expected in a system that has a frequency of 2.25 MHz, then the sample spacing should be 0.05 -inches or smaller.

The temporal sampling, in general, should be at least four times the center frequency of the transducer. So, greater than $20-\mathrm{MHz}$ sampling rate is recommended for a $5-\mathrm{MHz}$ transducer system. 


\subsection{DISCUSSIONS ON THE STRENGTHS AND LIMITATIONS OF SAFT-UT}

A fundamental concept to remember when implementing the SAFT techniques, is that a divergent beam is needed in the test material to fully utilize the focusing capabilities of the algorithm. With this in mind, then, one would intuitively expect the SAFT implementation to perform best on thicker material. In fact this is true. For very thin material (relative to wavelength) using a traditional focused probe technique may produce satisfactory results. Thin material can be classified as approximately less than 10 wavelengths in thickness. However, if the material has parallel and flat surfaces, SAFT may be implemented in a multiple bounce configuration; effectively generating thicker material to the system.

One may choose to perform SAFT processing on a given data set primarily to improve the image quality by taking advantage of the inherent spatial averaging that takes place in the coherent summation process.

The SAFT-UT utility set, in addition to providing a means for implementing SAFT techniques, provides a convenient and versatile set of tools for the archival and interpretation of NDE data sets. The data need not be limited to ultrasonic waveforms, and the signal processing need not necessarily be SAFT.

Currently there are no techniques implemented in this set of utilities to compensate for surface roughness, surface deviations, or material curvature.

The tandem SAFT techniques (TSAFT and TSAFT-2) assume that the near and far surfaces are flat and parallel. Deviations from this will cause distortions in the image: primarily positional in nature.

All coherent summation techniques implemented in this utility set assume homogeneous and isotropic material. Significant deviations from this assumption may produce unpredictable results. 


\subsection{SCANNING CONVENTIONS}

It is important to follow the scan conventions of the SAFT-UT utilities to properly utilize them. Figure 19 graphically shows the scan convention assumed throughout the utilities. The $Z$ axis represents depth into the material with the axis origin located at the specimen surface; the deeper into the material the larger the value of $Z$.

The $X$ axis is the scan axis at the surface of the material. The $Y$ axis is the increment axis. The scan mechanism is assumed to have:

1) traveled in the positive $X$ direction while collecting a plane of data,

2) incremented in the $Y$ direction,

3) scanned back to the original position of $x$ without collecting data, and

4) again traveled in the positive $x$ direction collecting the next plane of data.

It is essential that the data file A-scan records be ordered in this manner.

For angle beam applications, care must be taken to conform to the incident angle convention. Universally, the utilities expect the transducer to be tilted along the $X$ (scan) axis as shown in Figure 14. Also, if the transducer is placed so that it points in the direction of the scan (so the sound field precedes the transducer) then this is a negative incident angle. In most applications the incident angle will be negative.

If a foreign data file is to be used that is not structured in the format described, then it needs to be restructured to conform. Unpredictable results will occur if the processing and graphics utilities are implemented on a nonstandard data file. Three utilities have been written to assist in restructuring data files: SHUFFLEX, SHUFX, and SWAPXY. Consult the SAFT-UT Utilities REFERENCE MANUAL for further information about these utilities, and for further information related to adapting foreign data files to the SAFT-UT format consult the SAFT-UT Utilities INTERFACE AND INSTALLATION GUIDE. 


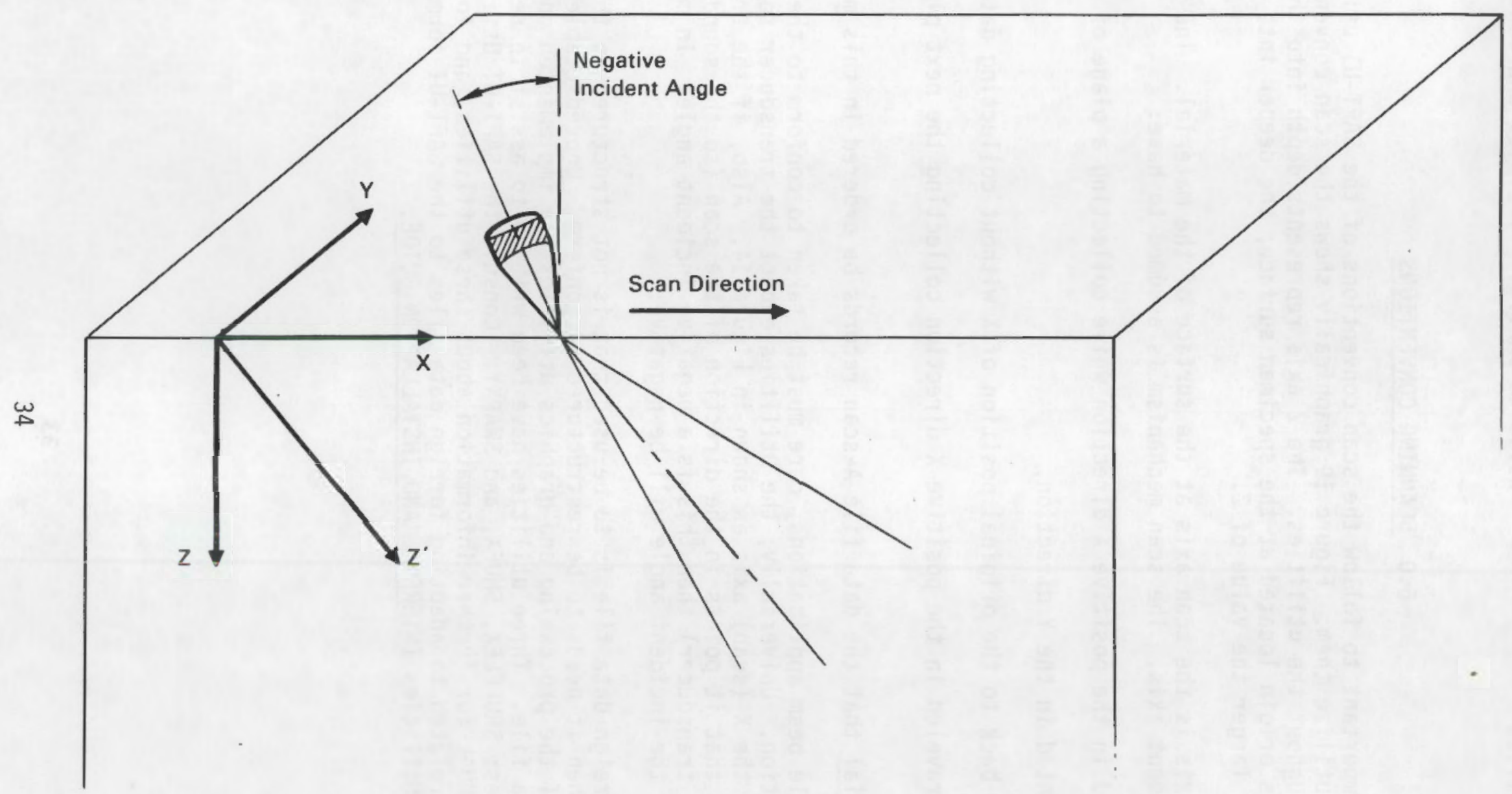

唄



FIGURE 19. SAFT-UT Scan Convention 


\subsection{WHERE TO GO FOR MORE INFORMATION (REFERENCES)}

This section is a reference base to aid the individuals who desire to discover more about SAFT technology than what is presented in this documentation set. Moreover, this section hopefully will assist those who desire to pursue new avenues using the robust SAFT algorithm. A reference list is provided with a primary emphasis of articles dated after 1983 with a few exceptions.

Norton's paper entitled "Theory of Acoustical Imaging" [5] supplies the basis for wide-band applications of SAFT-UT. It is a landmark paper that explains that traditional coherent summation is actually an approximation that holds only for narrow band applications. To perform wide-band SAFT one needs to perform the temporal derivative of the data file prior to processing the data.

The wide-band discussion is also explained by Busse, Collins, and Doctor in Section 3.3.3 of Review and Discussion of the Development of Synthetic Aperture Focusing Technique for Ultrasonic Testing [1]. The reference list included in Busse's paper includes further entries that date prior to 1983. This report has become a valuable reference document and is recommended reading for all serious users of the SAFT algorithm in ultrasonic applications. It provides a theoretical overview of fundamental SAFT techniques.

The paper by Langenberg [20] in the June 1986 NDT International magazine is a source for further references. This paper in particular references many in the European community. 


\section{REFERENCES}

1. Busse, L. J., H. D. Collins, and S. R. Doctor. 1984. Review and Discussion of the Development of Synthetic Aperture Focusing Technique for UTtrasonic Testing (SAFT-UT), U.S. Nuclear Regulatory Commission, NUREG/CR3625, PNL-4957.

2. Doctor, S. R., L. J. Busse, S. L. Crawford, T. E. Hall, R. P. Gribble, A. J. Baldwin, and L. P. Van Houten. 1986. Development and Validation of a Real-Time SAFT-UT System for the Inspection of Light Water Reactor Components, Semi-annual Report, April 1984 to September 1984, U.S. Nuclear Regulatory Commission, NUREG/CR-4583, PNL-5822, Vol. 1. Pacific Northwest Laboratory, Richland, Washington.

3. Doctor, S. R., T. E. Hall, L. D. Reid, S. L. Crawford, R. J. Littlefield, R. W. Gilbert. 1987. Development and Validation of a Real-Time SAFT-UT System for the Inspection of Light Water Reactor Components, Annual Report, October 1984 to September 1985, U.S. Nuclear Regulatory Commission, NUREG/CR-4583, PNL-5822, Vol. 2. Pacific Northwest Laboratory, Richland, Washington.

4. Doctor, S. R., T. E. Hall, L. D. Reid, G. A. Mart. 1987. Development and Validation of a Real-Time SAFT-UT System for the Inspection of Light Water Reactor Components, Annual Report, October 1985 to September 1986, U.S. Nuclear Regulatory Commission, NUREG/CR-4583, PNL-5822, Vol. 3. Pacific Northwest Laboratory, Richland, Washington.

5. Norton, S.J. 1976. Theory of Acoustic Imaging, Stanford Technical Report No. 4956-2.

6. Krautkramer, J., and H. Krautkramer. 1983 U1trasonic Testing of Materials, Third revised edition, Springer-Verlag, p 38.

7. Bracewell, R. 1965 The Fourier Transform and Its Applications McGrawHill, NY.

8. Ganapathy, S., B. Schmult, W.S. Wu, T.G. Dennehy, N. Moayeri, and P. Kelly. 1985 Design and Development of a Special Purpose SAFT System for Nondestructive Evaluation of Nuclear Reactor Vessels and Piping Components, Report No. NUREG/CR-4365, Nuclear Regulatory Commission, Washington, D.C.

9. Kino, G.S., D. Corl, S. Bennett, and K. Peterson, 1980. "Real Time Synthetic Aperture Imaging System." Ultrasonics Symposium Proceedings

Cat. \#80CH-1602-2S7, IEEE. 
10. Doctor, S. R., L. J. Busse, and H. D. Collins, 1983. "The SAFT-UT Technology Evolution," 6th International Conference on NDE in the Nuclear Industry, Zurich, Switzerland, pages 145-151, November 28-December 2, 1983.

11. Doctor, S. R., L. J. Busse, and H. D. Collins. 1984. "Development and Validation of a Real-Time SAFT-UT System for Inservice Inspection of LWRs," Proc. of the U.S. NRC Eleventh Water Reactor Safety Research Information Meeting, Vol. 4, NUREG/CP-0048, pages 65-80.

12. 8usse, L. J., H. D. Collins, and S. R. Doctor. 1984. "The Emerging Technology of Synthetic Aperture Focusing for Ultrasonic Testing," presented at 1984 Pressure Vessel and Piping Conference, San Antonio, Texas, 84-PUD-122, June 18-20, 1984.

13. Doctor, S. R., H. D. Collins, L. P. Van Houten, S. L. Crawford, T. E. Hall, A. J. Baldwin, R. E. Bowey, and R. P. Gribble. 1985. "Development and Validation of a Real-Time SAFT-UT System for Inservice Inspection of LWRs," Proc. of the U.S. NRC Twelfth Water Reactor Safety Research Information Meeting, Vol. 4, NUREG/CP-0058, pages 319-341.

14. Doctor, S. R., A. J. Baldwin, L. J. Busse, H. D. Collins, S. L. Crawford, and L. P. Van Houten. 1985. "SAFT-UT for Stainless Steel Pipe Inspection," Proc. of the 7 th International Conference on NDE in the Nuclear Industry, Grenoble, France, January 28-February 1, 1985.

15. Doctor, S. R., S. L. Crawford, T. E. Hall, and L. D. Reid. 1985. "SAFT Imaging in the Tandem Mode - TSAFT," ASNT Topical Meeting on Digital Signal Acquisition and Processing, Dallas, Texas, May 14-16, 1985.

16. Doctor, S. R., L. J. Busse, H. D. Collins, S. L. Crawford, and L. P. Van Houten. 1985. "Development and Validation of a Real-Time SAFT-UT System for Inservice Inspection of LWRs," Nuclear Engineering and Design, Vol. 86, pages 31-38, North Holland, Amsterdam.

17. Doctor, S. R., S. L. Crawford, and T. E. Hall. 1985. "SAFT-UT Field Experience," presented at 1985 Pressure Vessel and Piping Conference. New Orleans, Louisiana, PYP-Vol. 98-1, pages 203-210, June 22-26, 1985.

18. Doctor, S. R., T. E. Ha11, S. L. Crawford, and L. P. Van Houten. 1985. "Advanced Ultrasonic Imaging of Pipes in Dresden and Vermont Yankee Reactors," 8th International Conference on Structural Mecharics in Reactor Technology, Vol. C-D, August 19-23, 1985.

19. Doctor, S. R., T. E. Hall, and L. D. Reid. June 1986. "SAFT -The Evolution of a Signal Processing Technology for Ultrasonic Testing," NDT International, Vol. 19, No. 3, pages 163-167. 
20. Langenberg, K. J., M. Berger, Th. Kreutter, K. Mayer, V. Schmitz. June 1986. "Synthetic Aperture Focusing Technique Signal Processing," NDT International, Vol. 19, No. 3, pages 177-188.

21. Hal1, T. E., S. R. Doctor, and L. D. Reid. 1987. "A Real-Time SAFT System Applied to the Ultrasonic Inspection of Nuclear Reactor Components," Review of Progress in Quantitative Nondestructive Evaluation, Vol. 6A, Plenum Press, NY, pages 509-517.

22. Hall, T. E., S. R. Doctor, L. D. Reid, R. J. Littlefield, and R. W. Gilbert. 1987. "Implementation of a Real-Time U1trasonic SAFT System for Inspection of Nuclear Reactor Components," Acoustical Imaging, Vol. 15, Plenum Press, NY, pages 253-266.

23. Doctor, S. R., T. E. Hall, L. D. Reid, and G. A. Mart. 1986. "Application of Real-Time 3-D SAFT in the Tandem Mode," PNL-SA-14328, presented at the MPA Seminar, Stuttgart, West Germany, October 10, 1986.

24. Doctor, S. R., T. E. Hall, L. D. Reid, G. A. Mart, R. Littlefield, and R. Gilbert. 1986. "Development and Validation of a Real-Time SAFT-UT System for Inservice Inspection of LWRs," Proc. of Fourteenth Water Reactor Safety Information Meeting, Gaithersburg, Maryland, October 27-31, 1986, pages 19-41.

25. Doctor, S. R., T. E. Hall, and L. D. Reid. 1986. "Real-Time SAFT-UT for Nuclear Component Inspection," presented at the 8th International Conference on NDE in the Nuclear Industry, Orlando, Florida, November $17-20,1986$.

26. Doctor, S. R., T. E. Hall, L. D. Reid, and G. A. Mart. 1987. Development and Validation of a Real-Time SAFT-UT System for the Inspection of Light Water Reactor Components, Annual Report, October 1985-September 1986. NUREG/CR-4583, PNL-5822, Vol. 3. Pacific Northwest Laboratory, Richl and, Washington. 


\section{DISTRIBUTION}

No. of
Copies

OFFSITE

W. W. Shurtleff

Nondestructive Testing

Technology Division

Sandia National Laboratories

Albuquerque, NM 87185

4 C. Little

Nondestructive Testing

Technology Division

Sandia National Laboratories

Albuquerque, NM 87185

2 J. LaReau

Combustion Engineering, Inc. 1000 Prospect Hill Road

Windsor, CT 06095-0500

L. J. Busse

Aircraft Engine Business Group

General Electric Company

1 Neumann Way

Cincinnati, OH 45215-6301

S. L. Crawford

2337 Osborne Avenue

Santa Clara, CA 95050

2 DOE Technical Information Center
No. of

Copies

ONSITE

33 Pacific Northwest Laboratories

SR Doctor

TE Hall (20)

DK Lemon

GA Mart

RE Novak

GJ Posakony

LD Reid

Publishing Coordination (2)

Technical Report Files (5) 
